\title{
¿Un caso de "volver al futuro”?: Las Conclusiones sobre la Identificación del Derecho Internacional Consuetudinario de la Comisión de Derecho Internacional de la ONU*
}

\author{
A 'Back to the Future' Case? The International Law Commission Conclusions on Identification of Customary \\ International Law
}

\author{
Fabián Cárdenas ${ }^{a}$ \\ Universidad de Bogotá Jorge Tadeo Lozano, Colombia \\ fabian.cardenasc@utadeo.edu.co \\ ORCID: https://orcid.org/0000-0002-2601-1993
}

DOI: https://doi.org/10.11144/Javeriana.vj69.cvfc

Recibido: 21 Octubre 2019

Aceptado: 22 Enero 2020

Publicado: 21 Diciembre 2020

\section{Resumen:}

Este artículo sostiene que las conclusiones de 2018 sobre la identificación del derecho internacional consuetudinario producidas por la Comisión de Derecho Internacional de la ONU constituyen un retroceso conceptual de la costumbre a la perspectiva teórica de la fuente que se originó con el Estatuto de la Corte Permanente de Justicia Internacional de 1920, trayendo al presente las inconsistencias prácticas y teóricas del pasado. Aunque la Comisión tiene como propósito fundamental la codificación y el desarrollo del derecho internacional, este trabajo en particular resulta ser la expresión más anacrónica sobre la teorización de la costumbre internacional, pues retoma la perspectiva ortodoxa que casi cumple un centenario de existencia. Este artículo demuestra que estas conclusiones, lejos de ser un aporte novedoso, son una regresión teórica, la cual, gracias al esfuerzo de la Comisión, parecen estar a punto de volver a retomar vigencia. Ante tal situación, este artículo sostiene que, a pesar de los esfuerzos de la Comisión, no hay una teoría unívoca sobre la costumbre internacional, sino que, más bien, existen un conjunto de perspectivas teóricas que proponen posibles aproximaciones sin que ninguna de ellas pueda considerarse como dogma. Con ese propósito, este artículo contextualiza y analiza el proceso de consolidación de dicha perspectiva ortodoxa, luego presenta un conjunto de perspectivas alternativas que han teorizado sobre la fuente y, finalmente, presenta algunas críticas estructurales y específicas sobre las conclusiones de la Comisión lideradas por su relator, Sir. Michael Wood.

Palabras clave: Costumbre, Costumbre Internacional, Derecho Internacional Consuetudinario, Perspectiva Ortodoxa de la Costumbre, Derecho Internacional, Fuentes del Derecho Internacional, Práctica Estatal, Opinio Juris, Comisión de Derecho Internacional de la ONU.

\begin{abstract}
:
This article claims that the 2018 Conclusions on Identification of Customary International Law produced by the International Law Commission constitute a conceptual regression of customary international law to the theoretical approach originated in the 1920 Statute of the Permanent Court of International Justice, bringing back to the present the theoretical and practical inconsistencies of the past. Although the Commission has the fundamental purpose of codifying and developing international law, this particular work becomes the most anachronistic expression of the theory of customary international law. It goes back to the source's orthodox perspective, which by 2020 turns a century of existence. This research proves that the Conclusions, far from being a new approach, constitute a theoretical regression, which, thanks to the efforts of the Commission, seem to be in force again. In this vein, it is here sustained that there is no univocal theory on the identification of customary international law despite the Commission's pains. On the contrary, there are a series of theoretical perspectives proposing different possible approaches to the source, so that none of them can be considered dogma. With this purpose, the paper initially contextualizes and analyses the consolidation process of the orthodox perspective to customary international law, to later describe a set of alternative theoretical perspectives. Lastly, it presents some structural and specific critiques to the Commission's Conclusions led by the special rapporteur, Sir. Michael Wood.
\end{abstract}

Keywords: Custom, International Custom, Customary International Law, Orthodox Perspective of Customary International Law, International Law, Sources of International Law, State Practice, Opinio Juris, International Law Commission.

Notas de autor

a Autor de correspondencia. Correo electrónico: fabian.cardenasc@utadeo.edu.co 


\section{Introducción}

El 16 de junio de 1920 Léon Bourgeois, delegado del Consejo de la Liga de las Naciones, pronunció su discurso de bienvenida a los miembros del Comité Consultivo de Juristas en el Palacio de la Paz de La Haya. Sus palabras fueron las siguientes:

I welcome here Messrs. Adatci, Altamira, Bevilaqua, Loder, Lord Phillimore, Ricci Busatti and particularly Mr. Elihu Root, the great jurist and eminent American statesman, whose presence permits us to affirm that in the work which has called us together the old world and the new world, in spite of passing difficulties, cannot be separated by a durable barrier, and that indeed the two words "old world" and "new world" can now have no other meaning than the world of the past and the world of the future. For it is nothing less than humanity as whole which will form the new world of tomorrow. ${ }^{1}$

Así, el Estatuto de 1920 fue considerado la constitución del “mundo del futuro". En su discurso, Bourgeois separa el antes y el después del establecimiento de la Corte Permanente de Justicia Internacional (en adelante CPJI), para dibujar el límite entre el pasado y el futuro de la sociedad occidental. Para el Comité, el texto original Art. 38 y su nuevo catálogo de fuentes de derecho internacional, eran el "futuro" del nuevo orden mundial.

De acuerdo con esta visión de futuro, la CPJI traería consigo la modernidad y dejaría atrás el pasado. Es decir, contribuiría con el desmantelamiento del mandato opresor del derecho natural y la lucha por la supervivencia entre las naciones, detendría el despotismo y castigaría la tiranía del absolutismo, y, sobre todo, neutralizaría el antagonismo y la guerra generalizada. Las implicaciones de la modernidad jurídica ya han sido discutidas en otro momento ${ }^{2}$.

La intención de este artículo es demostrar que las Conclusiones sobre la Identificación del Derecho Internacional Consuetudinario son un retorno al "futuro" de 1920. En otras palabras, que los planteamientos de las Conclusiones de 2018, tal como fueron promovidas por el relator especial Wood, son anacrónicas y siguen dentro de los centenarios parámetros conceptuales que se comenzaron a establecer en el Estatuto de la CPJI que modela la ortodoxia. Además, como se podrá verificar adelante, se consolidan mientras que se ignoran de manera deliberada las múltiples perspectivas alternativas teóricas de la costumbre internacional (en adelante $\mathrm{CI})^{3}$, por atender criterios más políticos que jurídicos, como lo son el mantenimiento y posicionamiento de los dogmas que buscan perpetuar la tradición y el estatismo de la CI.

Para elaborar el argumento, este artículo se organiza en tres secciones. Primero, se traza y caracteriza la perspectiva ortodoxa de la costumbre internacional a la cual retorna la Comisión. Para esto se explora su génesis en las discusiones del Comité de Juristas, junto con su posterior desarrollo en la jurisprudencia de la Corte (CPJI-1920/CIJ-1945), haciendo especial mención de la introducción artificiosa de los dos elementos de práctica estatal y opinio juris, para más tarde poner en evidencia sus principales inconsistencias. Segundo, se propone una categorización de las perspectivas alternativas de la CI más sonadas, las cuales surgen desde los componentes tradicionales de la CI o desde otras fuentes que los trascienden. Tercero, se presentan una serie de argumentos para que apoyan la idea de "volver al futuro" por medio de la exposición de los dogmas de la perspectiva ortodoxa de la CI presente en el trabajo de la CDI. Finalmente se presentan algunas conclusiones.

\section{La perspectiva ortodoxa de la costumbre internacional}

En esta sección se identificarán los elementos que componen lo que denominamos la perspectiva ortodoxa de la costumbre internacional, o simplemente la ortodoxia ${ }^{4}$, la cual es la versión más apegada a los presupuestos teóricos tradicionales de dicha fuente. Una vez expuesta su construcción, postulados y naturaleza, presentaremos algunas de sus dificultades más resonadas. 
La ortodoxia está compuesta, por una parte, por el Art. 38 del Estatuto de la CPJI -reproducido posteriormente en el Estatuto de la CIJ-, el cual la define como la "prueba de una práctica generalmente aceptada como derecho"5. Complementariamente, está integrada por lo que se ha denominado la doctrina de los dos elementos (en adelante DDE) ${ }^{6}$, fabricada por la jurisprudencia de la Corte, y que sostiene que la CI emerge por la confluencia de práctica estatal -el elemento objetivo o material- y opino juris -el elemento subjetivo, psicológico o inmaterial- ${ }^{7}$. Contrario a lo que generalmente se cree, la ortodoxia no deviene del contenido literal del Art. 38, sino que es el producto de la construcción, adecuación y posicionamiento de la DDE, en la que la jurisprudencia de la Corte ha tenido un importante rol.

En la actualidad el Art. 38 es el punto de partida de un sin número de argumentos jurídicos internacionales y una inestimable cantidad de investigaciones en el campo. Ahora, a pesar de que solo es una norma en un tratado que regula un mecanismo de solución de controversias particular, goza de una indiscutible posición doctrinal, a tal punto que se ha considerado como la norma que enlista las fuentes del DI -la fuente de fuentes- ${ }^{8}$. Tal consolidación doctrinal, sin duda, se debe a que su naturaleza escrita da un parte de tranquilidad con la que sus antecesores nunca contaron, como es el caso de la International Prize Court, la cual nunca llegó a ser establecida9.

Yasuaki describe muy bien la posición que ocupa actualmente el Art. 38:

The failure to understand realistically the significance of the ICJ has influenced the attitude of international lawyers toward the question of the "sources" of international law. When discussing the problem of the "sources" of international law, most lawyers begin their argument by referring to Article 38 of the ICJ Statute. Even those who do not explicitly refer to Article 38 generally assume that discussion of the categories of international law should start with, the list of "sources" provided in Article 38(1). Although many leading international lawyers such as Jennings, Cheng, McDougal, Higgins, Falk and AbiSaab have recognized that using Article 38 for the purpose of explaining the categories of contemporary international law has an element of absurdity. ${ }^{10}$

Pese a que en la actualidad es discutible sostener categóricamente que el Art. 38 es la norma que regula las fuentes del DI, su importancia es innegable. La referencia ha sido utilizada indistintamente en convenciones y acuerdos arbitrales antes y después de 1945. Lo interesante es que la definición de CI contenida en el literal b ha tenido la misma suerte del artículo en su conjunto. Sin duda, esta situación ha sido reforzada por la propia Corte cuando ha afirmado que, desde un punto de vista superficial, su función primordial es resolver controversias entre los Estados por medio del derecho internacional a través del Art. 38, que brinda los elementos necesarios para el desarrollo de dicha tarea ${ }^{11}$.

Está claro que la CI no surgió del Art. 38, y pese a que el Estatuto incluye a la CI como una de las "fuentes formales" ${ }^{12}$ del DI, no la desarrolla conceptualmente y tampoco menciona en ningún momento los elementos tradicionales de opinio juris y práctica estatal, que posteriormente se manifestarían en la denominada DDE. Es posible que debido a esto la ortodoxia empezó a tener dificultades cuando tuvo que dar explicaciones sobre la CI, ya que sus argumentos se basaban en un origen dudoso, casi inexistente. A continuación, se discutirán el Art. 38 y la DDE de la CI.

\section{La génesis del Art. 38}

El texto del Art. 38 originalmente contenido en el Estatuto de la CPJI de 1920 fue propuesto por Barón Descamps ${ }^{13}$, quien fue el presidente del Comité Consultivo de Juristas que tuvo a cargo el establecimiento de la Corte. No obstante, a pesar de la notable importancia de las labores de este Comité colegiado, y pese a los diversos desacuerdos planteados en tanto que sus integrantes provenían de diversas familias jurídicas, la totalidad de las discusiones duraron apenas ocho días. Así, es debatible que dichos acuerdos constituyan 
hoy la base histórica, y para algunos dogmática, de las fuentes del derecho internacional y de la CI de forma particular.

En el Comité se plantearon tres posiciones diferentes respecto de la necesidad de definición de las fuentes. Por un lado, algunos juristas consideraron que el Estatuto debía dejar a criterio de los jueces cuáles serían las fuentes de derecho para utilizar en cada caso, y, por otro lado, otros consideraron que la intención del Estatuto era determinar con precisión la regla que la Corte debería implementar, al indicar tanto las fuentes como su contenido particular. Finalmente, una posición intermedia prevaleció según la cual el Art. 38 debería mencionar un conjunto de parámetros generales sobre la implementación de la norma, y, al mismo tiempo, entregó su desarrollo y aplicación al criterio de los jueces. Algunas de las posturas de los juristas servirán para ilustrar el debate.

Inicialmente, Root y Phillimore consideraron que definir tanto la CI como los principios generales implicaría caer en la completa petrificación. Consideraron que el comité no debía sobrepasar sus funciones debido a que su propósito era establecer la Corte más no indicarle qué normas aplicar en su ejercicio ${ }^{14}$. $\mathrm{Al}$ respecto, algunos de los miembros del Comité consideraron que dicha perspectiva impediría el desarrollo del DI, que, como es sabido, era muy joven en ese momento. Por otro lado, dejar parámetros laxos también sería contraproducente porque le otorgaría competencias prácticamente legislativas a la Corte, cuando son únicamente los Estados los que cuentan con dichas facultades ${ }^{15}$.

Loder y Descamps defendieron una visión más flexible del que sería el nuevo tribunal internacional. Ellos consideraban que el propósito del Comité era establecer los cimientos de una corte que con el tiempo pudiese crecer y desarrollarse. Contrario de poner obstáculos, estaban convencidos de que su deber consistía en crear mecanismos que fueran capaces de lidiar con la contingencia, al mismo tiempo que idear dispositivos jurídicos que le permitieran sobreponerse a posibles lagunas en las que los tratados no se hubieran pronunciado. De ese modo, un concepto general de la CI parecía ser el indicado desde su punto de vista ${ }^{16}$. Así mismo, Descamps pensó que limitarse a las normas existentes, es decir, a las normas escritas de aquel momento, como las normas exclusivamente aplicables, sería el equivalente de condenar la nueva corte a una pronta obsolescencia ${ }^{17}$.

El antagonismo entre las dos perspectivas, la que abogaba por la creación exhaustiva de reglas determinadas y la que proponía brindar amplias libertades al futuro tribunal (está de clara influencia anglosajona y aquella inspirada por el derecho continental europeo) derivó en el texto actual del Art. 38, el cual, en lo que tiene que ver con la CI, reconoce expresamente la existencia de dicha fuente al mismo tiempo que la deja intencionalmente indeterminada, de tal forma que a través de la entrada en funcionamiento de la Corte pudiera ser precisada y madurada ${ }^{18}$.

Actualmente, a pesar de que la CI se considera como una fuente dinámica e informal del $\mathrm{DI}^{19}$, críticas como las de Crawford no se han hecho esperar, cuando en perspectiva del texto actual sigue formulándose la pregunta How can the Court ever develop a rule that is customary? . $^{20}$.

A través de varias redacciones provisionales que dejaban un amplio rango de acción para la Corte ${ }^{21}$, y luego de algunas discusiones sostenidas en la Liga de las Naciones, finalmente el concepto de CI quedó plasmado en un texto amplio y confuso que llega a su centenario en 2020: "b. costumbre internacional como prueba de una práctica generalmente aceptada como derecho” ${ }^{\text {22 }}$. Más tarde, y sin ninguna discusión adicional, el mismo texto quedó en el Estatuto de la CIJ de 1945. Sin embargo, tal y como sostuvo Basdenvat, la razón por la que el texto prevaleció no fue precisamente por su éxito o aceptación, sino por la gran dificultad de hacer uno nuevo, sobre todo si se toman en cuenta la diversidad de intereses que estuvieron presentes en el Comité ${ }^{23}$. Por lo tanto, la simpleza de la cláusula desde el inicio se dejó en las manos de la Corte, esperando que esta pudiera dar un poco más de claridad a la ambigüedad conceptual original. 
Teniendo en cuenta que ni la redacción del Art. 38 ni los esfuerzos del comité que lo redactó incluyó en su conceptualización los elementos de práctica y opinio juris de forma explícita, podemos afirmar que existe una inconsistencia en la historia fundacional de la perspectiva ortodoxa, o, como lo precisa d'Aspremont, que su historia reposa en una falsa genealogía 24 .

Aunque el Art. 38 y su cláusula reconocen a la CI como una fuente del DI que se desarrolla a través de un conjunto de comportamientos sostenidos en el tiempo, no la describe o la explica en detalle. Esto, sin embargo, no es una excusa que absuelva las diferentes inconsistencias que más adelante serán presentadas ${ }^{25}$. Aun así, ello no quiere decir que se niegue por completo el importante servicio que ha prestado como guía. Al respecto, Pellet sostiene que el Art. 38 no merece ni elogios desmedidos ni ser condenado a la indignidad ${ }^{26}$; en sus palabras, podemos acercarnos a algunas de las reacciones que produjo en el siglo XX:

It can be seen as a superfluous and useless clause, at best a clumsy and outmoded attempt to define international law, at worst a corset paralyzing the world's highest judicial body. It can also be analyzed as a most successful and concise description of, both, the Court's mission and the law it must apply and as providing helpful guidance for avoiding non liquet as well as fantasy and arbitrariness in the interpretation and implementation of the rules of law. ${ }^{27}$

De este modo, es indiscutible que la CI ha sufrido la misma suerte que el Art. 38 en su conjunto. La cláusula, en el mejor de los casos, debe considerarse como la enunciación de un tipo de fuente cuyo contenido se ha dejado a disposición de la libre interpretación de la Corte. Luego de haber expuesto las endebles raíces del Art. 38 nos podemos hacer a una idea más clara sobre su naturaleza, lo que ayuda a disminuir la importancia sobredimensionada que se le ha dado al Estatuto, considerado usualmente como la aproximación regular a la $\mathrm{CI}^{28}$. A pesar del origen poco virtuoso de la redacción del Art. 38, sería injusto concluir que su importancia es nula porque pese a todas sus dificultades, se considera el cimiento de la jurisprudencia de la "Corte Mundial", en la cual se sustenta la ortodoxia, y, al mismo tiempo, la DDE de la CI. De este modo, puede afirmarse que, si el Estatuto dibujó el marco conceptual, la jurisprudencia de la Corte llenó los vacíos.

\section{La Doctrina de los Dos Elementos: la práctica estatal y la opinio juris}

Como se expuso, fue el Comité quien decidió deliberadamente dejar el contenido del Art. 38 aparentemente incompleto e indeterminado, pero en ningún momento mencionó de manera explícita el texto que la CI estuviera compuesta por uno u otro elemento. Entonces la DDE de la CI, cuyos componentes son la práctica estatal y la opinio juris, fue una invención de la jurisprudencia de la CPJI/CIJ y no un desarrollo conceptual contenido en el texto original o la voluntad inicial de los redactores.

Teniendo en mente la cláusula del Art. 38, Tams comenta que, si bien da pistas, no es mucho lo que aporta, aunque sin duda reconoce que se refiere a una práctica aceptada como derecho, aunque ello no quiere decir que ambas cosas deban considerarse como distintas, sino más bien como partes integrales de la definición de $\mathrm{CI}^{29}$. Además, agrega que en ningún punto el comité distingue tajantemente entre práctica estatal u opinio juris, o siquiera anticipó que tuvieran que existir alguno de dichos elementos para que existiera una norma consuetudinaria ${ }^{30}$. $\mathrm{Al}$ respecto, Haggenmacher señaló acertadamente que la cláusula "práctica general aceptada como derecho" sólo se refiere a uno de los componentes de la CI, lo que afirma, de nuevo, que la DDE de la CI es una creación posterior al Art. $38^{31}$.

La primera vez que la CPJI interpretó el Art. 38 a fin de sugerir la existencia de dos elementos fue en el Caso Lotus $^{32}$, a propósito del accidente entre los buques Bozkourt y Lotus, francés y turco respectivamente. Así, la Corte tuvo que pronunciarse sobre la posición francesa, según la cual la bandera que identifique al Estado cuya embarcación fuese acusada tiene la jurisdicción sobre la controversia penal surgida del incidente. Francia trató 
de demostrarle a la Corte una práctica en donde Estados diferentes de la nacionalidad de las embarcaciones tuvieron que abstenerse de iniciar procedimientos penales con el fin de comprobar su afirmación. Al respecto la Corte identificó por primera vez los elementos subjetivo y objetivo de la CI, al considerar que dichos casos:

$[\mathrm{M}]$ erely show that States had often, in practice, abstained from instituting criminal proceedings, and not that they recognized themselves as being obliged to do so; for only if such abstention were based on their being conscious of having a duty to abstain would it be possible to speak of custom. ${ }^{33}$

Frente a la discusión, algunos jueces no estuvieron de acuerdo con la postura mayoritaria y decidieron darle más peso a la evidencia de una práctica repetida con motivaciones consistentes a modo de deducir normas consuetudinarias, lo que desde su punto de vista daría balance a los dos componentes recientemente identificados ${ }^{34}$. Aquel fue el único escenario en donde la Corte discutió sobre la formación de la CI, luego de ello la Corte simplemente se ha limitado a afirmar que la práctica que sustenta una norma ha existido, sin detenerse mucho a demostrarlo ${ }^{35}$.

La referencia más reproducida sobre la DDE de la CI en la jurisprudencia de la Corte, y que además se considera como la referencia general sobre la fuente, es la desarrollada en el caso de la Plataforma Continental del Mar del Norte en donde se afirmó lo siguiente:

Two conditions must be fulfilled. Not only must the acts concerned amount to a settled practice, but they must also be such, or be carried out in such a way, as to be evidence of a belief that his practice is rendered obligatory by the existence of a rule of law requiring it. ${ }^{36}$

Más tarde, la Corte reconoció explícitamente la importancia de los dos elementos en su perspectiva. Esta sostuvo que la confirmación de una práctica estatal está directamente relacionada con la existencia de una opinio juris particular ${ }^{37}$. No obstante, aunque la Corte no se ha atenido a sus pronunciamientos, es innegable que ha contribuido constantemente a mantener la idea de que la CI es la suma de la práctica estatal y la opinio juris $^{38}$.

Describamos pues, según la perspectiva ortodoxa qué debemos entender por práctica y opinio juris. Por una parte, la ortodoxia usualmente entiende la práctica estatal como las diferentes conductas generales a través de las cuales el requerimiento objetivo de la CI es definido ${ }^{39}$, entre los cuales pueden ser incluidos la proclamación de normas internas, decretos de gobiernos nacionales, declaraciones frente a escenarios diplomáticos, comunicados de prensa, decisiones de tribunales y organizaciones internacionales ${ }^{40}$. Además, la práctica debe ser continua y uniforme por lo que el comportamiento contradictorio no puede contribuir a la formación de una norma consuetudinaria, tal y como quedó establecido desde el Caso del Asilo ${ }^{41}$. Del mismo modo, a pesar de que puede haber peticiones de costumbre instantánea ${ }^{42}$, la perspectiva ortodoxa requiere de un tiempo razonable antes de que una norma consuetudinaria pueda surgir, aunque el tiempo razonable pueda ser un siglo completo o un período corto de tiempo ${ }^{43}$. Por ejemplo, los aspectos esenciales del derecho internacional del mar fueron consolidados luego de cientos de años de práctica, sin embargo, las normas del derecho aeroespacial fueron consolidadas casi al instante luego de que los primeros satélites fueran lanzados a orbita. ${ }^{44}$.

Por otro lado, la opinio juris cive necessitatis usualmente es considerada como el componente subjetivo, o psicológico, de la CI; según la ortodoxia, es la creencia de que un determinado comportamiento estatal obedece al cumplimiento de una obligación jurídica preexistente ${ }^{45}$. Por lo tanto, de acuerdo con Dinstein, para identificar la opinio juris se debe prestar atención a las opiniones que expresan los sujetos internacionales en escenarios multilaterales, tales como la Asamblea General de las Naciones Unidas (AGNU) ${ }^{46}$. Como era 
de esperarse, la opinio juris ha sido el elemento más controversial de la DDE de la CI, porque su identificación depende de un requisito imposible de conseguir, como lo es identificar los pensamientos de un Estado, si es que esto puede hacerse de algún modo ${ }^{47}$.

Si evaluamos en perspectiva las intenciones iniciales del Comité al redactar el Art. 38 se podría concluir que, efectivamente, las mismas se cumplieron. Esto por cuanto no fue el Estatuto, sino la práctica de la Corte lo que llenó de contenido conceptual a la CI, solucionando, por un lado, la necesidad de consagrar una referencia escrita para que fuese respetado el principio de consentimiento de Estado, y, por el otro lado, la necesidad de aportar al desarrollo del DI. Así, se logró la aparente combinación perfecta entre objetividad y subjetividad, la cual se ha dado a lo largo de la utilización de la DDE en la doctrina de la Corte sobre la $\mathrm{CI}^{48}$. Sin duda, es la versión más extendida de la CI, ya que es constantemente repetida, y por lo tanto validada ${ }^{49}$, de tal modo es posible afirmar que, desde esa perspectiva, hay irrefutable evidencia de la CI donde están presentes dichos componentes $^{50}$.

\section{Las inconsistencias de la ortodoxia}

A pesar de que la Corte ha logrado erigir relativamente su perspectiva de la CI "como prueba de una práctica generalmente aceptada como derecho" ${ }^{51}$, es preciso resaltar que han sido muchos los obstáculos que ha encontrado en el camino, sobre todo por las inconsistencias y contradicciones presentes a lo largo de sus planteamientos. Esta sección identificará y describirá algunas de sus dificultades más evidentes, las cuales se categorizarán entre conceptuales, textuales, prácticas y probatorias.

\section{Inconsistencias conceptuales}

Para empezar, sus inconsistencias conceptuales tienen que ver con que, por un lado, se le ha criticado a la cláusula de ser excesivamente amplia, y, por lo tanto, incierta o indeterminada, y, por el otro, se ha comentado que el Estatuto entregó demasiada discrecionalidad a la Corte, lo que ha significado que solo se cuente con las declaraciones de la Corte en cada caso, y en consecuencia que no haya una teoría de la CI propiamente dicha.

Sobre la primera inconsistencia conviene tener en cuenta dos posiciones generales. Por una parte, Crawford

y Pellet consideran que el propósito original de la redacción era la generalidad y relativa indeterminación ${ }^{52}$. Por otra parte, Yasuaki sostiene que hay una inconsistencia conceptual cuando la definición ortodoxa se convierte en la aproximación dominante de la CI, al estar sustentada en una ecuación incorrecta entre normas de conducta y normas de adjudicación. Así, si bien el Art. 38 es solo una norma que regula el funcionamiento de un tribunal (adjudicación), no tiene por qué convertirse en el parámetro normativo de las fuentes (prescripción). Lo mismo ocurre cuando el DI es analizado como derecho doméstico, en donde ambos tipos de norma resultan confundiéndose ${ }^{53}$.

Yasuaki también sostiene que la jurisprudencia de la CIJ sobre la CI no puede considerarse como derecho internacional general ${ }^{54}$. Cómo ejemplo, se sugieren las Resoluciones de la $\mathrm{AGNU}^{55}$, porque, desde su punto de vista, se trata de normatividad que ha sido promocionada por Estados poderosos que no han tomado en cuenta la opinión de la gran mayoría de Estados del mundo ${ }^{56}$. Así, Yasuaki señala que esa situación de poder asimétrica se debe a la excesiva amplitud de la cláusula, la cual le ha permitido a la Corte desarrollar conceptos como aquiescencia, acuerdos tácitos o inferidos, los cuales han servido para prolongar dichas imposiciones ${ }^{57}$. 
Because the study of international law has been West-centric, the lack of state practice and opinio juris of a large number of non-Western nations has not been seriously considered [...] the fact that non-Westerners constitute the overwhelming majority of the human species has been ignored: first by the argument that they were not subjects of international law; and then, after the attainment of their independence, by the continued pre-eminence of the mythical theory of customary international law. ${ }^{58}$

Respecto de la discrecionalidad excesiva que se le dio a la Corte, Mendelson sostiene que debido a que a esta no le interesa compartir sus metodologías de trabajo, es muy difícil -sino imposible- deducir una teoría sobre el camino que toma para llegar a pronunciarse de una u otra forma respecto a la identificación de $\mathrm{CI}^{59}$. Ello revela que la Corte no da explicaciones sobre sus pronunciamientos y simplemente se limita a citar jurisprudencia en donde haya dicho con anterioridad que determinada conducta es considerada como CI. Tal situación ha generado la sensación de que el texto de la cláusula no es el que la Corte utiliza para hacer su trabajo, incluso aún y cuando está claramente determinado en su Estatuto ${ }^{60}$. Al respecto, Pellet se distancia arguyendo que esa complejidad es una característica propia tanto del derecho en general como de la CI en particular. Incluye que debido a que identificar una norma consuetudinaria está directamente relacionada con el conocimiento, la sensibilidad y la intuición de personas formadas en el derecho como un arte, no es posible entender la cuestión a través de una racionalidad objetiva de las ciencias naturales ${ }^{61}$.

\section{Inconsistencias textuales}

Las inconsistencias textuales orbitan alrededor de las distintas valoraciones que podrían hacerse de la cláusula "evidencia de una práctica generalmente aceptada como derecho"62, algunas de las cuales se han posicionado con respecto al énfasis dado a uno u otro componente de la DDE. Por un lado, están algunos autores que afirman que la redacción es defectuosa, por ejemplo, Waldock considera que, a pesar de mencionar los dos elementos, el orden en el que están escritos es equivocado, porque es la práctica la que evidencia a la costumbre y no viceversa ${ }^{63}$; es como tener los ingredientes adecuados, pero en el orden incorrecto. Lauterpacht, al contrario, sostiene que la costumbre no es una práctica que crea una norma jurídica, sino una conducta que antecede una norma jurídica preexistente ${ }^{64}$.

Oppenheim, por su parte, se ubica en un punto intermedio, ya que considera que los elementos deben aparecer concomitantemente, aunque menciona que no es coincidencia que la práctica estatal se mencione en primer lugar, ya que debe entenderse como la externalización de una convicción de que se actúa como si se tratara de una obligación consagrada en el derecho ${ }^{65}$. Por otro lado, algunos autores afirman que la redacción es apropiada, como Ferrari, por ejemplo, quien asegura que el problema no es la redacción, sino que la confusión teórica se debe a que hay una interpretación inadecuada del propósito inicial de la norma, porque, desde su punto de vista, el objetivo de la cláusula no era determinar la CI, sino establecer el modo por el que la Corte identificaría el contenido de las normas consuetudinarias ${ }^{66}$.

\section{Inconsistencias prácticas}

En la misma corriente, las inconsistencias prácticas están relacionados con que la formulación de la cláusula contiene una paradoja cronológica que, por un lado, es revisada y solucionada por medio de elementos exteriores a los que componen la CI, y, por el otro, es reconocida y superada con el fin de que la fuente siga funcionando con normalidad. Bradley describe dicha paradoja de la siguiente manera: 
If the states practices do not become binding as CIL until the states involved act out of a sense of legal obligation, how do the states develop that sense of legal obligation in the first place? In other words, the standard conception of CIL paradoxically seems to require that CIL develop before it can develop, in which case we would never have CIL. ${ }^{67}$

Entonces parece que el Art. 38 presta atención a las normas existentes y deja de lado el posible surgimiento de nuevas normas consuetudinarias. De modo que, mientras que una norma vigente puede ser evidenciada por medio del desarrollo de una práctica motivada por un sentido de obligación jurídica, parece que para este concepto es imposible crear nuevas normas ya que, a pesar de que el elemento de la práctica esté presente, nunca habrá opino juris. Esto se debe a que ningún sujeto actuará con la convicción de estar cumpliendo una obligación a menos de que la misma haya emergido como costumbre con anterioridad. En otras palabras, el origen de la CI nunca llegaría a darse.

$\mathrm{Al}$ respecto, hay quienes han considerado soluciones que implícitamente aceptan sus imprecisiones, de las cuales se pueden destacar tres. Primero, quienes sostienen que la opinio juris inicial está basada en un error, donde muchos sujetos jurídicos equivocadamente creyeron que determinada acción era requerida por la ley;

algo parecido a lo que sostiene la teoría del error de derecho ${ }^{68}$. Segundo, quienes sostienen que se debe a que un número pequeño de Estados acogen una norma consuetudinaria, aunque no consideren que es requerida por una costumbre preexistente, y otros Estados la aceptan y se acogen a lo que haya sido determinado con antelación; o como Bradley la llama, Fiat theory ${ }^{69}$. Tercero, quienes consideran que el problema se debe a una concepción equivocada de la opinio juris, entre los cuales se encuentra Lepard, quien sostiene que la opino juris no requiere de la creencia de que haya una ley que dependa de determinada conducta, sino que es suficiente con que haya un acuerdo generalizado de que una norma en particular es necesaria ${ }^{70}$. Ahora, también hay quienes reconocen dicha paradoja, pero no le dan mucha trascendencia, entre los cuales están el US Restatement y la CDI, quienes sostienen que no tiene implicaciones prácticas importantes y que las imprecisiones en la formulación han prevenido parálisis de la $\mathrm{CI}^{71}$. Tal muestra de tolerancia respecto a las impresiones de la cláusula es un motivo para creer que no se consideran muy importantes para el desarrollo del CI en la cotidianidad.

\section{Inconsistencias probatorias}

Finalmente, y no por ello menos importante, las inconsistencias probatorias tienen que ver con que no se han establecido acuerdos sobre aspectos fundamentales, como, por ejemplo, cuáles son las cantidades necesarias de los elementos para que haya una costumbre, cuántos Estados deben haber estado involucrados en el proceso de formación, cuál es el criterio para saber si las declaraciones diplomáticas pueden ser consideradas como opinio juris, entre otros. Es por ello por lo que consideramos que la CI se da por medio, casi que exclusivamente, de una interpretación subjetiva de los dos componentes desarrollados por la DDE que propone la ortodoxia.

De este modo, nótese que el concepto de costumbre como prueba de una práctica generalmente aceptada como derecho, que para el año 2020 cumple un siglo de existencia, no solo tiene una genealogía confusa, sino que está minada de múltiples inconsistencias que inician con su inadecuada redacción, pero que se agravan con las inconsistencias lógicas, prácticas y probatorias que anteriormente se exponen. Sin duda, esto la configura como un recurso poco fiable para comprender la CI en el derecho internacional contemporáneo. 


\section{Perspectivas alternativas de la costumbre internacional}

En esta sección se busca poner de presente las diversas perspectivas que han surgido en la teoría jurídica internacional como alternativas ante las diversas dificultades e inconsistencias de la perspectiva ortodoxa de la CI.

$\mathrm{Al}$ contrario de lo que sostienen las miradas tradicionales, aquí se afirma que no hay una sola manera de explicar la CI, lo que quiere decir que, por muy elaborada que sea una u otra teoría, no hay una sola que logre describir completamente su complejidad, o, en otras palabras, ninguna teoría es completamente correcta o incorrecta. De esa manera, aquí se postula que no hay una norma univoca que regule la totalidad de la CI, sino que hay un conjunto de perspectivas teóricas que brindan elementos que permiten construir una idea de lo que es la CI. Lo que quiere decir que el entendimiento que se tenga sobre dicha fuente siempre estará mediado por la aceptación del conjunto de planteamientos de determinada perspectiva.

Además, es importante resaltar que pese a las creencias generalizadas -que suelen esparcirse por cuenta de los manuales de DI-, la ortodoxia no representa necesariamente la "doctrina mayoritaria". A pesar de que durante mucho tiempo se consideró como la única manera de interpretar la CI, hoy en día las perspectivas alternativas han venido abriéndose paso en los debates sobre la fuente, a tal punto que, en la actualidad, en la doctrina especializada, las perspectivas alternativas resultan ser más dominantes que el mismo discurso de práctica y opinio juris de la ortodoxia. De este modo, si bien la perspectiva ortodoxa es utilizada formalmente por Estados y tribunales internacionales, las perspectivas alternativas abundan en la literatura teórica jurídica ${ }^{72}$.

No obstante, no es el propósito de este artículo hacer una lista completa de todas las perspectivas teóricas alternativas disponibles, sino más bien dar un panorama teórico comprensible que permita tener en cuenta algunas de las aproximaciones más importantes.

Para tales efectos, está sección presenta algunas aproximaciones teóricas que podemos agrupar en, por un lado, las que parten de la DDE, es decir, del énfasis en la práctica estatal o en la opinio juris, y, por el otro, de otros puntos que no son contemplados por esta, tal como las miradas del derecho doméstico, los procesos y las relaciones internacionales.

Como se expondrá, en muchos casos, las alternativas teóricas varían dependiendo el punto de vista o el énfasis teórico desde los cuales se plantee la perspectiva o el esfuerzo de conceptualización de la CI. Así, los resultados variarán dependiendo si se parte desde una mirada dogmática, si existe un especial enfoque en el elemento práctico o psicológico, si se compara con el derecho doméstico, si hay un enfoque en el proceso más que en el resultado, o si hay una influencia ideológica evidente, como partir desde las creencias de las escuelas críticas del derecho internacional, entre otros.

\section{Desde la Dogmática}

Esta es la perspectiva más divulgada en la gran mayoría de los manuales generales de derecho internacional alrededor del mundo. Esta se funda en la existencia de dogmas, teniendo la perspectiva ortodoxa de práctica estatal y opinio juris como uno de ellos. La dogmática no se preocupa por explicar, desarrollar o entender la perspectiva ortodoxa; simplemente la da por sentada por el simple hecho de ser la postura oficial, tanto de la Corte como de la CDI. Por ende, su teoría consiste en evadir la necesidad de teorizar al simplemente aceptar los lineamientos de las perspectivas con mayor autoridad.

Posicionarse en la ortodoxia es más una elección política que jurídica. Quienes la acogen aceptan dogmas que determinan su naturaleza, no cuestionan ni critican la evidencia de su inconveniencia. Tampoco toman en cuenta los planteamientos que han explicado a la CI desde uno de los elementos de la DDE o desde otras 
perspectivas. Por lo tanto, se limitan acogerla irrestrictamente y a ejercerla tal y como ha sido desarrollada desde la tradición.

Quienes se posicionan desde la ortodoxia son herederos de la tradición del DI europeo, los cuales pueden ser ejemplificados en el Manual de Derecho Internacional Público de Max Sørensen o el Brownlie’s Principles of Public International Law, entre muchos otros reconocidos textos jurídicos.

Los autores de dichas obras no dudan en afirmar que la ortodoxia ha sido la única perspectiva con la que el sistema jurídico internacional ha contado, al destacar que se ha desempeñado satisfactoriamente para lo que inicialmente fue propuesta. Esto se debe, en parte, a que ellos mismos han sido quienes de uno u otro modo, directa o indirectamente, han contribuido a darle forma y a incrementar su legitimidad en múltiples escenarios, tanto académicos como institucionales.

\section{Desde la práctica}

Estos escépticos consideran imposible descifrar la mente de un Estado u otro sujeto de DI. Para ellos es imposible saber lo que piensa un Estado, aunque si es posible evidenciar lo que dice que piensa. Esta perspectiva pone todo su énfasis en la práctica estatal, la CI es únicamente lo que se puede ver en la realidad, nada más debe ser tomado en cuenta para determinar su existencia. Una de las más claras representaciones de esta perspectiva es la que dejó en 2000 la Asociación de Derecho Internacional (en adelante ILA, por sus siglas en inglés:

A customary international law is one which is created and sustained by the constant and uniform practice of States and other subjects of international law in or impinging upon the international legal relations, in circumstances which give rise to the legitimate expectation of similar conduct in the future. ${ }^{73}$

Como podemos ver la ILA evita completamente utilizar la opinio juris, creencias, o pensamientos con el fin de insistir que sólo a través de la práctica se puede tener una expresión del componente inmaterial, el cual es entendido como la expectativa de que en el futuro se dé una conducta similar ${ }^{74}$. Así la conducta y la práctica son las que exclusivamente determinan la existencia de una norma consuetudinaria. D'Amato, quien se acoge y defiende esta perspectiva, sostiene su escepticismo de que siempre se habla de un componente material que realmente no existe porque siempre es determinado por otros factores ${ }^{75}$.

Para D’Amato resulta más importante preguntarse por el medio que rodea a la CI que por los asuntos que exploran su naturaleza. Al plantearse preguntas como: ¿por qué es necesaria la CI? o ¿por qué apareció en primer lugar la CI? podemos hacernos a una idea sobre las posibles soluciones a todas sus inconsistencias. D'Amato considera al sistema internacional como un actor que debe ser tomado en cuenta para estudiar a dichos fenómenos en su conjunto ${ }^{76}$, y en donde dicho sistema tiene principalmente dos metas: la paz y la maximización del bienestar ${ }^{77}$, dado que las controversias son el resultado del trabajo para cumplir con dichas metas, las cuales terminan afectando la escogencia de las normas de la CI. Por lo tanto, para D’Amato la CI es un conjunto de reglas vigentes que se desempeñan como una maquinaria de resolución de controversias.

Ahora, existen otros autores que se acogen a lo anterior sin desconocer que existen otros factores pueden afectar la configuración de la CI. Kelsen, por ejemplo, quien por mucho tiempo ha sido mal interpretado, es uno de los autores más representativos de esta postura ${ }^{78}$.

En los años 30, Kelsen describió la CI como un hábito regular que va a acompañado por la convicción de acarrear una violación de la ley en caso de incumplimiento ${ }^{79}$. Así, Kelsen reconoce la necesidad de una convicción de estar actuando de acuerdo con el derecho para que exista la CI. Su perspectiva destaca el componente material sin negar el inmaterial, así este le parezca inconveniente. Más tarde en 1953, explica que la convicción de que se está actuando o absteniéndose de actuar de acuerdo con el derecho es la esencia sin la 
cual no puede hablarse de CI. De otra manera, solo se podría hablar de un uso, debido a que dicha práctica sostenida en el tiempo no genera ni pretende generar obligaciones jurídicas.

Otros ejemplos pueden ser, por un lado, Tunkin, quien señala que el la CI es un tipo de norma internacional que se forma a través de la práctica estatal, y que solamente alcanza su desarrollo en el momento en el que las actuaciones repetidas de los Estados son validadas por medio de la coordinación de sus voluntades, lo que

gradualmente termina por convertir en obligatorias a dichas actuaciones ${ }^{80}$. Por otro lado, Mendelson, quien no habla de coordinación de voluntades, sino de la expectativa de comportamientos similares, sostiene que la CI surge y es sostenida por prácticas constantes y uniformes tanto de Estados como de sujetos de derecho internacional, en donde por medio de sus relaciones, se va configurando la expectativa de conductas similares en el futuro ${ }^{81}$.

\section{Desde el elemento psicológico}

Está perspectiva considera que la práctica, o cualquier forma de externalización del derecho es solo una consecuencia de los deseos más profundos del Estado. Así, esta perspectiva asume que los Estados y los sujetos internacionales tienen alma o corazón. Por lo tanto, esta perspectiva sostiene que para que exista CI es necesaria únicamente la opino juris, es decir, hace énfasis en el elemento subjetivo o inmaterial. La idea de norma no escrita de Hugo Grotius es el mejor ejemplo, tal y como lo señala en The Law of the Nations: “[...] a primary rule, or first principle, the spirit of which is self-evident and immutable [...] God Himself says this spellbind through the voice of nature $[\ldots]^{382}$, y agrega, "[...] when any right is given to any one in such a way that it is not taken away from anyone else, then it is called custom" $"$.

Esta perspectiva le da la potencia de la inmanencia a la CI, de modo que ni siquiera una práctica constante y continua puede derogar la fuerza avasallante del derecho; y, es que, en esos términos, ¿quién puede discutir con Dios? ${ }^{84}$. Así, la validez de cualquier planteamiento de la CI reposa en su correspondencia con el derecho de las naciones, que no fue construido por medio de las relaciones sociales, sino que simplemente le fue entregado a los hombres. Es por eso que quienes se acogen a esta perspectiva naturalista están más interesados en crear medios para establecer las evidencias, que en la formación de la CI. Aunque para algunos las ideas de Grotius sean una cosa del pasado, sus posturas siguen presentes a través de autores más modernos como Verdross ${ }^{85}$. Ha sido tal su influencia que la Corte ha reconocido la existencia de la consciencia común de la humanidad. Lo mismo ha sido notorio en ejemplos como los juicios de Nuremberg, el Preámbulo de la Declaración de los Derecho Humanos, en el caso de Aplicación de la Convención de Prevención y Castigo al Crimen de Genocidio $^{86}$, entre otros.

Curiosamente, Koskenniemi reconoce desde su perspectiva crítica la existencia del concepto de consciencia colectiva de la humanidad, lo que tiene que ver con que la CI no sea el producto de un consentimiento explícito, sino más bien el resultado de una conciencia jurídica colectiva del mundo civilizado ${ }^{87}$. No obstante, dicha conciencia no está exenta de las relaciones de poder, debido a que es la tentativa coronada por el éxito de las civilizaciones occidentales de imponer su visión del mundo sobre el resto de las civilizaciones. No obstante, su visión no llega al extremo del realismo, sino que entiende que en el derecho internacional puede haber balance debido a que la maleabilidad de la costumbre le permite unas posibilidades estratégicas, tanto para el poder como para la resistencia ${ }^{88}$.

En esa misma corriente, Lepard redefinió la opinio juris a través de presupuestos éticos ${ }^{89}$, señalando que es un requisito según el cual los Estados consideran que es deseable, ahora o en un futuro cercano, la existencia de un principio legal o norma prescriptiva, que permita o prohíba determinada práctica estatal ${ }^{90}$. Así, aunque 
no niega la existencia de la practica estatal, la relega a ser una evidencia de la opinio juris, siendo esta la única condición para que emerja la $\mathrm{CI}^{91}$. Ahora, desde una posición más moderada, Bernhardt concluyó que en situaciones excepcionales no hace falta que haya práctica estatal para que surja una CI, siempre y cuando haya sido reconocida y aprobada universalmente, lo que balancea la presencia de los elementos necesarios por medio de la particularidad del contexto ${ }^{92}$. Brownlie, quien se acoge a lo anterior, agrega que la cantidad de un componente -opinio juris o práctica- depende del campo específico en donde se esté desarrollando la norma consuetudinaria, lo que quiere decir que no hay una norma unívoca que regule la $\mathrm{CI}$, sino que depende de las características del contexto en el que transite ${ }^{93}$.

Por nuestra parte, en otro espacio hemos sostenido que, desde el punto de vista de la ortodoxia, las dinámicas contemporáneas del DI han generado una disminución en la relevancia del elemento material en pro de un notable incremento en el peso que tiene el elemento subjetivo. De modo que en contextos disciplinares como los derechos humanos o el derecho internacional ambiental ha sido posible el surgimiento y consolidación de normas consuetudinarias únicamente por cuenta de la convicción de obligatoriedad de las mismas y aún a pesar de práctica opuesta ${ }^{94}$. No obstante, es precisamente el propósito de este artículo desarrollar dicho argumento.

\section{Desde la lógica del derecho interno}

Esta perspectiva explica la CI por medio de los planteamientos con los que cuenta el derecho interno para comprender el derecho consuetudinario, o en general, todo el derecho no escrito ${ }^{95}$. Como es de esperarse, los resultados de las explicaciones difieren de acuerdo con la tradición jurídica en donde tenga lugar, razón por la que se hace necesario consultar tanto la tradición del Derecho Continental Europeo como la del Common Law.

Por un lado, desde el Common Law, hay autores que reconocen la existencia de la consciencia colectiva de la humanidad, pero lejos de ser inmanente, destacan que es una construcción colectiva, y, por lo tanto, desde su perspectiva, la CI debería ser tratada como si fuera un Common Law Internacional ${ }^{96}$. Waldock, por ejemplo, señala que ambas comparten la misma naturaleza no escrita, la cual funciona como una fuente debido a la esencia descentralizada de la comunidad internacional. Así, el origen de las normas consuetudinarias es diverso, es decir, niega la existencia de una única norma que rija la CI. A veces surgen de la interacción de los sujetos internacionales, a veces surgen de las tradiciones teóricas y jurídicas, lo que hace de la CI una especie de folclor jurídico internacional, no muy lejos de lo planteado por Koskenniemi ${ }^{97}$.

Por el otro lado, Thirlway señala la cercanía conceptual del significado general de la costumbre como una fuente jurídica de diferentes sistemas de tradición civil, y, aunque la esencia de la conceptualización sea similar, cada uno de los planteamientos difiere de acuerdo con las características de cada país. De esa manera, por ejemplo, mientras que en Francia la llamada coutume d'origine savante reposa en las tradiciones jurídicas y en las máximas más que en la práctica de quienes están regidos por el derecho ${ }^{98}$, en Alemania la Gewohnheitsrecht es considerada como una fuente teórica del derecho que está basada en la práctica de sus jueces (Richterrecht). Thirlway considera que la importancia actual de la CI ha disminuido en la práctica de los sistemas jurídicos modernos domésticos, pero sigue siendo una fuente muy importante del derecho internacional ${ }^{99}$ 


\section{Desde el proceso}

Esta perspectiva sostiene que la CI es el producto de un proceso, independientemente de la consideración de los componentes de la DDE; lo único que comparte con los planteamientos anteriores es que no hay un acuerdo sobre sus elementos. En esa medida, Hart define a la CI como normas que surgen a través de un proceso lento de crecimiento, en donde las conductas dejan de ser opcionales para convertirse en habituales y luego en obligatorias ${ }^{100}$. Su posición contiende con la de Cheng, quien considera que es posible que haya costumbres instantáneas al considerar que la formación de una norma de CI siempre requerirá tiempo y repetición $^{101}$. Así, aunque pareciera que Hart toma a la práctica como el centro de su planteamiento, se enfoca más en el camino hacia la consecución de la norma consuetudinaria que en el fin en sí mismo y los elementos conceptuales que lo componen ${ }^{102}$. Por lo demás, su conceptualización debe ser leída junto con su interpretación del rol de los jueces, el cual es, en su opinión, el criterio determinante que hace posible el surgimiento de la CI.

Treves toma el mismo camino que Hart, evadiendo una conceptualización precisa y optando por ubicar a la CI en lo que él denomina como un silogismo cacofónico, debido a que es un proceso que es, al mismo tiempo, la consecuencia de un proceso ${ }^{103}$. De la misma manera, d'Aspremont señala la inevitable informalidad de la perspectiva ortodoxa y los riesgos derivados de ello ${ }^{104}$, y, probablemente inspirado en Hart, nos brinda un concepto más general y amplio de la CI como “(...) the embodiment of these rules which are ascertained short of any written instrument” ${ }^{\prime 105}$, en donde además destaca la necesidad de que los componentes arriben concomitantemente, en un periodo de tiempo que permita que dicho proceso genere un resultado ${ }^{106}$.

Aun así, para d'Aspremont la perspectiva basada en el proceso no alivia el peligroso carácter informal tanto de la identificación como de la evidencia de la CI, lo cual solo puede ser corregido por medio del trabajo de los jueces, como lo planteó Hart, pues son ellos quienes pueden dar cimientos formales a la teoría de la CI. Así, es evidente el interés por encontrar un criterio de identificación formal para distinguir entre lo que es y no es CI más allá de los dos elementos de la ortodoxia, puesto que siempre están cubiertos por un denso manto de indeterminación que termina minando su autoridad normativa ${ }^{107}$.

\section{Desde las escuelas críticas del derecho internacional}

Está perspectiva asocia a la CI directamente con el poder, como lo señala d'Aspremont, la CI es una "pista de baile", un concepto maleable que permite impulsar todo tipo de agendas, tanto personales como colectivas, que busca hacerse a los medios para ejercer el poder de acuerdo a una voluntad particular, y que incluso pueden llegar a ser contradictorias entre sí. Entonces, la CI es un instrumento de poder, o como lo sostiene Kabblers, el inevitable reflejo del poder ${ }^{108}$. Por ello es por lo que Worster considera que sin importar cual sea el concepto de CI siempre será construido con la finalidad de satisfacer intereses particulares ${ }^{109}$. Además, en ese orden de ideas, el planteamiento de Kelly según el cual la CI es construida deductivamente a partir de los pronunciamientos de actores poderosos ${ }^{110}$ no parece del todo descabellada, lo que, entre otras cosas, implica un cuestionamiento directo a la compatibilidad de la CI con la legitimidad y la autoridad democrática.

Algunos autores no caen en el negativismo y sostienen que es importante tener confianza en las autoridades que identifican y aplican la CI. Por ello se han enfrentado a duros cuestionamientos, entre los cuales están los de Jennings, quien les contesta que lo que ellos se esfuerzan insistentemente en llamar CI no solo no es CI, sino que nada tienen que ver con la $\mathrm{CI}^{111}$. Tal vez por ese tipo de posiciones vehementes es que Fidler la llama 
"la perspectiva peligrosa", por lo que se asocia directamente a la posibilidad de abusar del poder, lo que no es de extrañarse si se toma en cuenta su definición de la CI, según la cual es el equivalente a una lista de deseos para Navidad en el derecho internacional ${ }^{112}$.

Esta perspectiva tiene tantos matices como autores, quienes beben del realismo y de los estudios críticos del derecho internacional, donde, por ejemplo, se encuentran Yasuaki y Klabbers, quienes consideran que las posibilidades que permite la CI no están a disposición de todo el mundo, sino que han sido concentradas y están dispuestas a la voluntad de un grupo selecto de países occidentales ${ }^{113}$. Al mismo tiempo, Reisman, en representación de la escuela de New Haven, reconoce el rol de la CI como una catapulta del poder, la cual ha sido utilizada por países del norte global como una estrategia ante la pérdida de las mayorías en los escenarios democráticos en donde los países del sur estaban empezando a negociar con sus propias agendas ${ }^{114}$. Por lo tanto, su definición de la CI es fiel al espíritu de esta perspectiva, pues considera que es un término vago que dice muy poco de normas importantes que no pasan un proceso legislativo, y que, debido a ello, son parte de estrategias de manipulación en una democracia de por si debilitada, en donde algunos construyen un derecho internacional a su conveniencia sin entregar nada a cambio ${ }^{115}$.

No es gratuito que algunos de los autores consideren que un regreso a la CI sea parte de una estrategia política internacional, de tal manera que parece que lo que más les incomoda no es el hecho de que la CI sea un instrumento del poder, sino que aquellos que se benefician de ello, de una u otra manera, sean hipócritas y no lo reconozcan cuando son expuestos.

Ahora, teniendo en mente este conjunto de perspectivas alternativas, no es posible afirmar que haya una sola manera de interpretar la CI. Todo lo contrario, existe un abanico de posibilidades, múltiples aproximaciones teóricas que explican su identificación. Cabe señalar que ninguna es completamente acertada o errada, pero todas se erigen de acuerdo o en contra de la perspectiva ortodoxa. Finalmente, lo anterior implica que hay diversidad sin completa fragmentación teórica.

\section{"Volver al futuro": el trabajo de la Comisión de Derecho Internacional de la ONU}

En 2012 la CDI designó a Sir Michael Wood como relator especial para la identificación del derecho internacional consuetudinario. Luego de la presentación de cinco reportes y dos memorándums de la secretaria $^{116}$, en los que, sin lugar a duda, se recopiló una gran cantidad de insumos sobre la CI de múltiples fuentes de la más alta calidad, en 2018 fueron aprobadas finalmente las "Conclusiones sobre la identificación de la costumbre internacional” ${ }^{117}$.

Ahora, es oportuno destacar que esta sección no tiene por objetivo analizar exhaustivamente cada uno de los debates y materiales que fueron incluidos en el trabajo del relator especial. Por el contrario, presenta las evidencias de porqué se considera que las Conclusiones son una regresión conceptual a la perspectiva ortodoxa. En otras palabras, que las conclusiones posicionan los dogmas de la ortodoxia por encima de las perspectivas alternativas de la CI, tal vez con el propósito de actualizar los medios por los cuales se ha impuesto dicha visión de la CI en el DI.

De esa manera, presentaremos algunas de sus críticas estructurales, y, al mismo tiempo, iremos mencionando sus principales desafíos, tal y como fueron expuestos en la primera sección. Cabe aclarar que consideramos que son estructurales porque son decisiones que afectan la totalidad de lo planteado por las conclusiones, es decir, son elecciones que delimitan y condicionan su posible alcance, teniendo en cuenta que la tarea fundamental de a la CDI es codificar la vanguardia teórica del DI. 
La primera crítica estructural es que las conclusiones dan por sentado que el Art. 38 del Estatuto de la Corte es el referente necesario de la CI. Utiliza el contenido de la cláusula, el cual enlista las fuentes del DI como la referencia escrita sobre la CI. No comenta, por ejemplo, que es únicamente un artículo en un tratado sobre un mecanismo de resolución de controversias, que no fue escrito con el propósito de regular o describir con detalle la CI o que ha sido calificado como excesivamente amplio e indeterminado. Simplemente se acogió irrestrictamente a lo planteado por la tradición, y, en ese momento, dejó sentado lo que consideramos es el primer dogma contenido en la ortodoxia, como lo es que la prevalencia del Art. 38 como el origen de la CI es una elección política más que jurídica, que es un presupuesto que no se justifica ni se comprueba, y, por lo tanto, es el primer paso para que las conclusiones sean consideradas como parte de la perspectiva ortodoxa.

La segunda crítica estructural es que las conclusiones asumen que en la redacción del Art. 38 están presentes los dos elementos constituyentes de la CI, a saber: la práctica estatal y la opinio juris ${ }^{118}$. No obstante, como se explicó en la primera sección, los dos elementos fueron el resultado de la interpretación que hizo la Corte sobre la CI a través de su jurisprudencia, tal y como había sido propuesto por los autores del Estatuto a comienzos del siglo XX, lo que quiere decir que no solo estaban ausentes en el planteamiento original, sino que son una invención artificial, la cual no puede ser extraída de la redacción del Art. 38 propiamente dicha.

Así, consideramos que lo anterior constituye el segundo dogma contenido en la ortodoxia. Tal y como se puede ver en las conclusiones, quienes se acogen a sus planteamientos, es decir, a todos los autores que incluimos en la perspectiva teórica que se apega a la dogmática ven y afirman vehementemente que en la redacción del Art. 38 están presentes los dos elementos de la DDE. Por lo tanto, al igual que en la crítica anterior, no se debate ni se cuestiona la evidencia que hemos presentado, sino que se asume como una verdad absoluta, es decir, permítasenos la redundancia, que la ortodoxia está constituida por dogmas que hallan su expresión más reciente en las conclusiones.

La tercera crítica estructural es que las conclusiones ignoran la inexistencia de una regla secundaria unívoca que regule la CI, así como las perspectivas teóricas alternativas que la pretenden describir, las cuales, curiosamente, son reconocidas de algún modo en el primer reporte de Wood. Así pues, se decide arbitrariamente acoger la DDE como la metodología para identificar a la CI. Nótese que el planteamiento básico de la DDE que consiste en determinar "whether is a general practice, and whether such general practice is accepted as law (that is, accompained by opinio juris" "19 , es originalmente decidido, más no discutido. De modo que las conclusiones y todo el trabajo de la Comisión gira en torno a la comprensión detallada de los componentes internos de la DDE, cuando la selección de dicha doctrina o perspectiva no fue discutida, sino simplemente impuesta. En otras palabras, el trabajo de la Comisión abrió el debate ante la comunidad internacional con respecto a la comprensión de la práctica y la opinio juris, así como su interacción. Sin embargo, la selección de esta DDE fue simplemente dada por cierta, asumida como un dogma, cuando lo cierto es que la misma solo es una de muchas perspectivas teóricas posibles que ha planteado la doctrina jurídica internacional.

Aunque desde el Primer Informe de 2012 el relator especial había señalado que la DDE era la metodología que la gran mayoría de Estados había utilizado para identificar a la CI en muchas ocasiones ${ }^{120}$, la elección propiamente dicha solo se dio hasta el Segundo Informe de 2013, debido a que, según su interpretación de las discusiones, todos los miembros del Comisión estuvieron de acuerdo y dieron bienvenida a la metodología, siempre y cuando se lograran establecer medios para conocer la relación apropiada entre la práctica estatal y la opinio juris ${ }^{121}$.

La elección de la DDE como la metodología para la identificación de la CI implicó necesariamente que toda investigación tuviera que asumir tanto sus aciertos como sus defectos, lo que consideramos que es la configuración del tercer dogma presente en las conclusiones. Desde ese mismo momento, todos los esfuerzos del relator especial estarían condicionados a investigar única y exclusivamente dentro de los linderos de la perspectiva ortodoxa y, si tenemos en cuenta que dicha perspectiva data de comienzos del siglo XX y que 
el trabajo del relator especial comenzó en 2012, no podemos sino señalar que dicha elección condenó a cualquiera que fuera el resultado de su trabajo a ser calificado de anacrónico, lo cual hubiese podido ser evitado, entre otros caminos, por medio del reconocimiento de la indeterminación de la DDE o del desacuerdo en torno a la acentuación de los elementos como tal.

Ahora, una vez escogida dicha metodología el trabajo del relator especial se centró en describir detalladamente la práctica estatal y la opinio juris, para lo cual utilizó un conjunto de requerimientos que se deben usar para filtrar las evidencias que aspiren a ser consideradas como parte de una CI. Antes de profundizar en lo último, cabe mencionar que según las conclusiones la presencia de uno solo de los elementos no basta para la identificación de una CI, porque, desde su punto de vista, una práctica estatal sin opino juris, incluso si es consistente y general, no es más que un comportamiento sin ningún tipo de fuerza vinculante, y ocurriría lo mismo del lado de la opino juris ${ }^{122}$.

$\mathrm{Al}$ respecto, la posición de Wood sobre las perspectivas alternativas y su apego a la perspectiva ortodoxa son evidentes cuando afirma que:

"While writers have from time to time sought to devise alternative approaches to the identification of customary international law, emphasizing one constituent element over the other or even excluding one element altogether, such theories have not been adopted by States or in the case law". ${ }^{123}$

Consideramos que los requerimientos establecidos por las conclusiones son un intento de dar claridad a las impresiones de la perspectiva ortodoxa y que, a pesar de que el relator especial se esfuerza por dar los mejores ejemplos y explicaciones, parecen ser únicamente un sofisticado armazón conceptual detrás del cual perdura un conjunto de planteamientos teóricos que buscan insistir en una perspectiva dogmática, como lo es la DDE de la CI. A continuación, presentamos algunas razones que explican por qué no pueden considerarse como novedosas las conclusiones a la luz de las inconsistencias planteadas en la primera sección. Lo que, a su vez, demuestra porqué el trabajo de la Comisión en materia de CI es un evidente caso de "Volver al Futuro", o a lo que la Comisión de Juristas de 1920 consideraba el futuro del derecho internacional en ese entonces.

La conclusión tres señala que para que una práctica sea considerada como CI se debe tener en cuenta, primero, la duración de la practica en el tiempo, y, segundo, el grado de generalidad en relación a su extensión, representatividad y constancia. No obstante, advierte que en cada caso concreto puede llegar a variar ${ }^{124}$.

Como ya lo mencionamos, no existe una duración determinada que deba demostrarse para que se dé el surgimiento de una costumbre, lo que se ilustra, por ejemplo, en el momento en el que la CIJ consideró que en el caso de la Plataforma Continental del Mar del Norte no importaba que no hubiese pasado poco tiempo, con la condición de que la práctica hubiese sido extensiva y uniforme ${ }^{125}$.

De la misma manera, a pesar de que es sabido que el número de Estados debe ser suficiente para que se configure una CI, no existe una regla o un número que determine con presión cuántos Estados son suficientes, a lo que la CIJ y la CDI han hecho frente por medio de la representatividad, es decir que el requisito se cumple en el momento en el que los Estados que se ven particularmente afectados por la CI participan activamente en su configuración ${ }^{126}$.

La constancia, entendida como el establecimiento de determinado patrón de conductas por parte de los Estados, debe ser sostenida en el tiempo en el mejor de los casos. Aunque no debe ser perfectamente constante para que emerja una costumbre y tampoco se puede decir que es establecida unívocamente. Así, la Corte ha adoptado tanto posiciones estrictas como laxas al respecto, en donde, por ejemplo, en el caso Plataformas del Mar del Norte exigió una práctica "extensiva y virtualmente uniforme", mientras que en el caso Nicaragua se posicionó de manera más laxa, bastando que la conducta de los Estados fuera consistente "en general” ${ }^{127}$.

Así mismo, la conclusión nueve señala que el elemento material en sí mismo es insuficiente para que surja una costumbre, y, por lo tanto, siempre debe ir acompañado del componente psicológico como lo sostiene la ortodoxia. De esa manera, si tomamos la definición de opinio juris de la CIJ, "creencia de que [una] práctica 
resulta obligatoria por la existencia de una regla de derecho que la requiera” ${ }^{128}$, la cual es aceptada y respalda indirectamente por las conclusiones, encontramos que ninguna de sus proposiciones es novedosa, puesto que se limita los mismos términos de la doctrina ortodoxa.

En uno de los comentarios la CDI afirma lo siguiente:

"It is thus crucial to establish, in each case, that States have acted in a certain way because they felt or believed themselves legally compelled or entitled to do so by reason of a rule of customary international law: they must have pursued the practice as a matter of right, or submitted to it as a matter of obligation". ${ }^{129}$

Aquí se hace evidente que sigue estando presente la noción de "creencias subjetivas de los Estados", y, por lo tanto, las conclusiones continúan negando el hecho de que son los funcionarios estatales los que actúan a través del Estado con sus propias voluntades individuales. De esa manera, el requisito probatorio del elemento psicológico se sigue dando por medio de la demostración de los pensamientos o creencias del Estado.

$\mathrm{Si}$ las conclusiones sostienen que los Estados piensan, encontrar los medios por los cuales expresan sus motivaciones es una tarea muy importante. Es por ello por lo que la conclusión diez presenta un conjunto de formas de evidencia del elemento psicológico, que, aunque no es exhaustivo, toma en cuenta, por ejemplo, las declaraciones públicas de los Estados, las opiniones jurídicas gubernamentales, la correspondencia diplomática, entre otras.

En esa medida, quien considere que determinada manifestación es una expresión de la voluntad estatal debe presentar toda la evidencia que justifique porqué debe entenderse de tal manera. Esto significa que el planteamiento de las conclusiones no descifra los desafíos probatorios de la ortodoxia, puesto que sigue sin precisar los criterios que son tomados en cuenta en el momento de reconocer una expresión como evidencia del elemento inmaterial de la CI. Sin lugar a dudas, estas imprecisiones dan fuerza a los argumentos de las perspectivas alternativas según las cuales existen muchos motivos para creer que la CI siempre es determinada por medios subjetivos cualquiera que sea el caso.

Al mismo tiempo, si nos apartamos de lo que hacen los Estados y nos concentramos en lo que dejan de hacer, es decir, en sus abstenciones de obrar de cierta manera, que también se pueden llegar a configurar en una costumbre, siempre y cuando cumplan con lo que la CPJI en el caso Lotus determinó como "un deber consciente de abstenerse" ${ }^{\$ 130}$, las conclusiones tienen que enfrentar los desafíos que tienen que ver con la aquiescencia y las formas de consentimiento tácito que configuran en algunos casos la CI.

La figura del objetor persistente que es presentada en las conclusiones no resuelve lo que mencionamos en las inconsistencias textuales, puesto que mantiene las condiciones para que perduren las relaciones asimétricas de poder entre los Estados al no tomar en cuenta que los Estados se abstienen de reaccionar ante determinado acto por muchas razones distintas a la aprobación tácita, bien sea por no contar con las capacidades institucionales o financieras, o por no contar con suficiente preparación política o diplomática. Así se mantiene la sensación de que algunas normas consuetudinarias pueden emerger incluso sin contar con la aprobación efectiva de la mayoría de los Estados de la comunidad internacional.

Los ejemplos que hemos venido exponiendo hasta el momento son la evidencia de que, por un lado, la CDI acoge abiertamente la ortodoxia como su perspectiva de la CI, por otro lado, de que no está cumpliendo con su objetivo original, como lo es la codificación de los avances más importantes del DI. Por lo tanto, las conclusiones no aportan nada novedoso a la teorización de la CI, debido a al amplio cuestionamiento de la DDE de la CI por medio de perspectivas teóricas alternativas que, de uno u otro modo, han logrado dar con algunas precisiones. Aun así, no fueron tomadas en cuenta por las conclusiones.

Por lo tanto, que las conclusiones hayan escogido la DDE significa que no han titubeado en dar el primer paso para reivindicar la ortodoxia como la perspectiva imperante del órgano más influyente a nivel mundial sobre la teorización del DI, y, por lo tanto, de la CI.

En este punto, y teniendo en cuenta todo lo anterior, conviene preguntarnos sobre la legitimidad de las conclusiones, es decir, ¿quiénes respaldaron o no el resultado del trabajo del relator especial? Ello puede verse 
representado en los comentarios de los gobiernos ${ }^{131}$, donde se encuentran los insumos que cada uno de los Estados hizo llegar al relator especial para contribuir en el modelamiento de las conclusiones, y, si se presta atención a quienes son los interlocutores y cuáles son los intereses que defienden, encontraremos que de los escasos veintiún países que participaron -casi un diez por ciento de los Estados del mundo- solo seis se pueden

considerar del sur global, y de cuales solo dos fueron de América Latina ${ }^{132}$. Así fueron en su mayoría países del norte global los que incidieron en su configuración, en los cuales destacan, por ejemplo, Estados Unidos, Reino Unido, China, Rusia y Alemania.

Ante tal panorama valdría preguntarse: ¿cómo se debería interpretar la prevalencia de unos y la relativa ausencia de otros frente a la determinación de los parámetros identificadores de la CI? La respuesta se encuentra en los comentarios que varios Estados enviaron como contribución a la tarea del relator, en los cuales se pueden identificar dos tendencias. Por un lado, los países del norte global, los cuales aportan copiosas experiencias en lo que tiene que ver con el uso y la determinación de la fuente. Por otro lado, los países del sur global, que reconocen que no han tenido participación en la configuración de la fuente, y, que, por lo tanto, no es mucho lo que pueden aportar a la labor del relator especial. No obstante, reconocen la importancia de la fuente y su estatus en el $\mathrm{DI}^{133}$

Vale la pena formular varias inquietudes relativas tanto respecto del rol universalizador de la Comisión como de la lógica interna de la visión relativa a la identificación de la CI, ¿cómo debemos interpretar el silencio de todos los países que no participaron en el proceso de configuración de las conclusiones?

Como ya tenemos presente, debemos tomar en cuenta las distintas posiciones que se pueden dar frente al asunto, como, por ejemplo, ¿el silencio de algunos de los países del norte global debe ser interpretado como aprobación o como desinterés?, o, por otra parte, ¿a qué se debe la escasa participación de los países del sur global?, ¿es cuestión de completa aprobación o desinterés o se debe más a la prudencia de la inexperiencia?, y, más importante aún, ¿están listos nuestros países para dar ese tipo de discusiones?, o, ¿debemos limitarnos a observar cómo son debatidos sus contenidos por aquellos que han intervenido en su historia? Finalmente, ¿son las conclusiones un esfuerzo por traer de vuelta a la actualidad a la ortodoxia con el fin de imponer su visión funcional al status quo para perpetuar las relaciones entre el norte y el sur global que se han dado históricamente en la comunidad internacional?

Resulta evidente que la ortodoxia se sustenta en dogmas que tienen como fuente una interpretación unívoca del Estatuto de la CPJI y la jurisprudencia de la Corte, la cual ha sido inmune a los cuestionamientos que se le han planteado desde las perspectivas alternativas de la CI. Por ello, las conclusiones son una expresión de la interpretación dogmática y, por lo tanto, ortodoxa de la CI. Esto puede explicarse si los planteamientos que sustentan la posición del relator especial se entienden más como una estrategia argumentativa de carácter político, que como una decisión involuntaria en el marco de una investigación sobre la CI. ${ }^{134}$

\section{Conclusiones}

Teniendo en cuenta el origen y evolución de la ortodoxia, sus diversas dificultades conceptuales y prácticas, la existencia de una variedad de teorías alternativas, y el complicado proceso llevado a cabo al interior de la CDI para producir las conclusiones sobre la identificación del derecho internacional consuetudinario, puede concluirse que la Costumbre Internacional no es el producto de opinio juris y práctica. Esto, muy a pesar de la existencia del discurso conceptual que repetitiva y mecánicamente suelen incluir los manuales tradicionales de derecho internacional.

Aunque la CDI hace un importante esfuerzo -no sin dificultades- al tratar de explicar los contenidos de la doctrina de los dos elementos, es importante resaltar que la elección de esta como punto de partida, obedece 
a una decisión metodológica -y política- con efectos jurídicos que no fue discutida, sino simplemente impuesta.

Esto trae consigo la relativización de la supuesta existencia de una regla secundaria unívoca que regule la costumbre en el derecho internacional. Así, la regla es que no hay regla. Por el contrario, tal y como lo acepta, más no lo incluye la CDI, tiene una diversidad de teorías explicativas de la CI, que, desde diversas aproximaciones, tratan de explicar su identificación y formación. En este orden de ideas, la DDE, reencauchada por el trabajo de la CDI a partir de la interpretación que hizo la CPJI/CIJ del Art. 38 de su Estatuto, resulta ser solamente una de muchas posibilidades argumentativas para explicar la CI, sin desconocer que es, sin duda, la de mayor visibilidad en la doctrina internacionalista.

Así mismo, las diversas dificultades que se han planteado en este artículo evidencian la necesidad de que los efectos del trabajo de la CDI en materia de CI sean tomados con más mesura, atendiendo no sólo a los problemas lógicos internos de la DDE, sino también a la relativa legitimidad de las conclusiones. Aunque Wood y la Comisión han dado por sentado el respaldo universal a su trabajo, no resulta de poca importancia la escasa participación de Estados en el proceso, en el que el protagonismo del minoritario norte global es evidente.

Aunque valdría la pena hacer un análisis por regiones, es importante tomar nota a manera de ejemplo el caso de Latinoamérica. De toda la región apenas se vio una tímida participación de dos Estados, con algunas comunicaciones escritas enviadas por El Salvador y alguna participación oral realizada por Uruguay. Pero ¿a qué se debe esto? ¿debemos interpretar la ausencia como beneplácito? Esa ha sido la conveniente interpretación de quienes lideraron el trabajo. Pero erealmente el silencio Latinoamericano constituye un guiño absoluto a la DDE? Darle estos excesivos alcances al silencio es desmesurado y perjudicial.

Sin embargo, como ya lo hemos justificado más ampliamente en otros espacios ${ }^{135}$, esta indeterminación conceptual debe asumirse no como excusa para caer en el absoluto escepticismo en el que suelen caer los críticos, sino como una oportunidad para la región y para el sur global en general, que les permita participar en la construcción comunitaria ${ }^{136}$ de una perspectiva argumentativa de identificación de la CI que también tenga en consideración los intereses propios y no se limite a propender de manera exclusiva los del norte global.

Abandonar la estrategia del silencio y de la indiferencia resulta necesario, toda vez que en la identificación de la CI reposan importantes intereses nacionales, tal como ocurre con el Estado colombiano, quien, para citar un ejemplo, se encuentra enfrentando una importante controversia jurídica contra Nicaragua ante la CIJ en la que están en juego vitales espacios marítimos y de soberanía. La adjudicación depende de manera directa de la decisión que tome la Corte respecto de la naturaleza de CI de una norma de la Convemar, como lo es la plataforma continental más allá de las 200 millas náuticas.

De este modo, nuestros Estados latinoamericanos, así como los del sur global, tienen ante sí la posibilidad de tomar parte en la construcción comunitaria argumentativa de los criterios de identificación de la costumbre en el derecho internacional, o de, con su silencio, allanar el camino para que la teoría jurídica de la CI pueda "volver al futuro" conceptual, consistente en una verdadera regresión y estancamiento teórico que ha promovido la CDI con sus recientes conclusiones.

\section{Bibliografía}

Alain Pellet, The Statute of the International Court of Justice: A Commentary (Andreas Zimmermann, Christian Tomuschat \& Karin Oellers-Frahm eds., Oxford University Press, 2006). http://pellet.actu.com/wp-content/u ploads/2016/02/PELLET-2006-Article-38-of-the-Statute-of-the-ICJ.pdf

Alfred Verdross, Les Principes Généraux du Droit dans la Jurisprudence Internationale, 52 Collected Courses of The Hague Academy of International Law (The Hague Academy of International Law,1935). 
Fabián Cárdenas. ¿Un caso de “volver al futuro” ?: Las Conclusiones sobre la Identificación...

Anthony D’Amato, Trashing Customary International Law, 81 American Journal of International Law, 101-105 (1987). https://doi.org/10.2307/2202136

Anthony D’Amato, Customary International Law: A Reformulation, 4 International Legal Theory, n. ${ }^{\circ}$ 1, 1-6 (1998).

Anthony D'Amato, New Approaches to Customary International Law, 105 American Journal of International Law, n. 1, 163-170 (2011). https://doi.org/10.5305/amerjintelaw.105.1.0163

Arzt Gunther, Ignorance or Mistake of Law, 24 American Journal of Comparative Law, n. ${ }^{\circ}$ 4, 646-679 (1976). https: //doi.org/10.2307/839579

Asamblea General de las Naciones Unidas, Reporte A/CN.4/663 (2013). https://undocs.org/pdf?symbol=es/A/C N.4/663

Asamblea General de las Naciones Unidas, Reporte A/CN.4/672* (2014). https://undocs.org/pdf?symbol=es/A/C N.4/672

Asamblea General de las Naciones Unidas, Reporte A/CN.4/682 (2015). https://undocs.org/pdf?symbol=es/A/C N.4/682

Asamblea General de las Naciones Unidas, Reporte A/CN.4/695 (2016). https://undocs.org/pdf?symbol=es/A/C N.4/695

Asamblea General de las Naciones Unidas, Reporte A/CN.4/717 (2018). https://undocs.org/pdf?symbol=es/A/C N.4/717

Asamblea General de las Naciones Unidas, memorándums A/CN.4/659 (2013). https://undocs.org/sp/A/CN.4/659

Asamblea General de las Naciones Unidas, memorándums A/CN.4/691 (2016). https://undocs.org/sp/A/CN.4/691

Asamblea General de las Naciones Unidas, memorándums A/CN.4/710/Rev.1 (2019). https://undocs.org/pdf?sym bol=es/A/CN.4/710/Rev.1

Dan Being, Mistake of Law, 30 Israel Law Review, n. ${ }^{\circ}$ 1-2, 214-222 (1996). https://doi.org/10.1017/S0021223700 015065

Bin Cheng, United Nations Resolutions on Outer Space: "Instant" International Customary Law? 5 Indian Journal of International Law, 23-112 (1965). https://doi.org/10.1093/acprof:oso/9780198257301.003.0007

Curtis Bradley, A State Preference Account of Customary International Law Adjudication,SSRN (2014). https://pape rs.ssrn.com/sol3/papers.cfm?abstract_id=2508298

Brian D. Lepard, Customary International Law: A New Theory with Practical Applications (Cambridge University Press, 2010). https://doi.org/10.1017/CBO9780511804717

Caso Accordance with International Law of the Unilateral Declaration of Independence in Respect of Kosovo, ICJ, Advisory Opinion, ICJ Reports (2010). https://www.icj-cij.org/en/case/141

Caso del Asilo (Colombia vs. Peru), ICJ, Advisory Opinion, ICJ Reports (1950). https://www.icj-cij.org/en/case/7

Caso Fisheries (UK vs. Norway), ICJ, Merits, ICJ Reports (1951). https://www.icj-cij.org/en/case/5

Caso Military and Paramilitary Activities in and against Nicaragua (Nicaragua vs. USA), ICJ, Judgment, ICJ Reports (1986). https://www.icj-cij.org/files/case-related/70/070-19860627-JUD-01-00-EN.pdf

Caso Jurisdictional Immunities of the State (Germany vs. Italy, Greece Intervening), ICJ, ICJ Reports (2012). https ://www.icj-cij.org/en/case/143

Caso de la Plataforma Continental del Norte (Alemania vs. Dinamarca, Países Bajos), ICJ, Merits, ICJ Reports (1969). https://www.dipublico.org/cij/doc/44.pdf

Caso Legality of the Threat or Use of Nuclear Weapons, ICJ, Advisory Opinion, ICJ, Reports (1996). https://www .icj-cij.org/en/case/95

Caso del S.S. Lotus (France vs. Turkey), ICJ, Judgment, PCIJ, Series A, No. 10 (1927). https://www.icj-cij.org/files/ permanent-court-of-international-justice/serie_A/A_10/30_Lotus_Arret.pdf

Casos del North Sea Continental Shelf (Federal Republic of Germany vs. Denmark, Netherlands), ICJ, Merits, ICJ Reports (1969). https://www.icj-cij.org/files/case-related/51/051-19690220-JUD-01-00-EN.pdf 
Caso Rights of Nationals of the USA in Morocco (France vs. US), ICJ, Merits, ICJ Reports (1952). https://www.ic j-cij.org/en/case/11

Caso Right of Passage over Indian Territory (Portugal vs. India), ICJ, Merits, ICJ Reports (1960). https://www.icjcij.org/en/case/32

Caso de S.S. Wimbledon (UK, France, Italy, Japan vs. Germany), Merits, PCIJ, Series A, No. 1 (1923). http://www.w orldcourts.com/pcij/eng/decisions/1923.08.17_wimbledon.htm

Caso Serbian Loans, PCIJ, Judgement, Series A, Nos. 20/21 (1929). http://www.worldcourts.com/pcij/eng/decisio ns/1929.07.12_payment1.htm

Comisión de Derecho Internacional de la ONU, Second report on identification of customary international law, A/ CN.4/672 (2014). https://legal.un.org/ilc/documentation/spanish/a_cn4_672.pdf

Comisión de Derecho Internacional de la ONU, Draft conclusions on identification of customary international law, with commentaries, A/73/10 (2018). https://undocs.org/en/A/73/10

Comisión de Derecho Internacional de la ONU, CDI, Identification of customary international law. A/RES/73/203 (2019). https://undocs.org/en/A/RES/73/203

Christian J. Tams, The International Court as a "Law-Formative Agency", en The Development of International Law by the International Court of Justice (Christian J. Tams and James Sloan eds., Oxford University Press, 2013). htt ps://doi.org/10.1093/acprof:oso/9780199653218.003.0016

Daniel Harold Levine, The Chronological Paradox in Customary International Law or, the Virtue of Sloopy Timing in a Messy World, (2005) (Ph.D. Dissertation, Georgetown University). https://drum.lib.umd.edu/bitstream/ha ndle/1903/7974/levine_dissertation.pdf?sequence $=1$

Danilo Zolo, Hans Kelsen: International Peace through International Law, 9 European Journal of International Law, Issue Vol. 9, 206-324, (1998).

David Fidler, Challenging the Classical Concept of Custom: Perspectives on the Future of Customary International Law, German Yearbook of International Law, Vol. 39, 198, (1996).

Declaración Universal de los Derechos Humanos, París, (1948).

Diego Mejía-Lemus, Some Considerations regarding “Instant International Customary Law”, Fifty Years Later, Indian Journal of International Law, 55, (2015).

Diego Uribe \& Fabián Cárdenas, Derecho Internacional Ambiental, Universidad Jorge Tadeo Lozano, (2010).

Estatuto de la Corte Internacional de Justicia, (1945).

Fabián Cárdenas, A Call for Rethinking the Sources of International Law: Soft Law and the Other Side of the Coin, Anuario Mexicano de Derecho Internacional, XIII, 355-403, (2013).

Fabián Cárdenas. Epilogue: International Litigation and the Stamp of Post-modernity en The Law of International Litigation: Legal Tools and Strategy for International Disputes Resolution in the 21 Century, 457-475 (Rafael Prieto Sanjuán ed., Pontificia Universidad Javeriana \& Editorial Ibáñez, 2015). https://www.academia.edu/150 21906/International_Litigation_and_the_Stamp_of_Postmodernity

Fabián Cárdenas \& Oscar Casallas, A Large Cup of Opinio Juris and Practice to Taste: The Recipe of Contemporary Customary International Law? 8 Colombian Yearbook of International Law, 87-130 (2015). https://doi.org/1 0.12804/acdi8.1.2015.03

Fabián Cárdenas, Customary International Law as an Argumentative Framework (2016) (tesis doctoral, Pontificia Universidad Javeriana). https://www.academia.edu/28227982/Customary_International_Law_as_an_Argum entative_Framework_An_Alternative_Theoretical_Approach_in_International_Environmental_Law

Fabián Cárdenas, Custom as the product of successful argumentative campaigns, en International Organizations, NonState Actors and the Formation of Customary International Law (S. Droubi \& J. d'Aspremont eds., Manchester University Press, 2020). https://doi.org/10.2139/ssrn.3545441

Fabián Cárdenas \& Jean d'Aspremont, Epistemic Communities in International Adjudication, en Max Planck Encyclopedia of International Procedural Law (2020). https://opil.ouplaw.com/view/10.1093/law-mpeipro/e 2425.013.2425/law-mpeipro-e2425?rskey=A8LDF9\&result=1\&prd=OPIL 
Fabián Cárdenas \& Santiago Rojas, Costumbre internacional: una lectura desde Latinoamérica a la perspectiva ortodoxa de la fuente no escrita del derecho internacional, en Manual de Derecho Internacional Público (René Urueña ed., Universidad de Los Andes, 2020).

François Rigaux, Hans Kelsen on International Law, 9 European Journal of International Law, n. 2, 325-343 (1998). https://doi.org/10.1093/ejil/9.2.325

Grigory Tunkin, International Law in the International System, 147 Collected Courses of The Hague Academy of International Law (1975). https://referenceworks.brillonline.com/entries/the-hague-academy-collected-course s/*A9789028610088_01

Hersch Lauterpacht, Règles Générales du Droit de la Paix, 62 Collected Courses of The Hague Academy of International Law (1937). https://referenceworks.brillonline.com/entries/the-hague-academy-collected-course s/*A9789028609921_02

Helbert Hart, The Concept of Law (2.. ed., Clarendon Press, 1994).

Hugh Thirlway, Concepts, Principles, Rules and Analogies: International and Municipal Legal Reasoning, 294 Collected Courses of The Hague Academy of International Law (2002). https://referenceworks.brillonline.com/entries/t he-hague-academy-collected-courses/*A9789041118561_04

Hugh Thirlway, The Sources of International Law (Oxford University Press, 2014).

Hugo Grotius, The Freedom of the Seas: Or the Right Which Belongs to the Dutch to Take Part in the East Indian Trade (Oxford University Press, 1916).

Humphrey Waldock, General Course on Public International Law, 106 Collected Courses of The Hague Academy of International Law (1962). https://referenceworks.brillonline.com/entries/the-hague-academy-collected-course s/*A9789028614321_01

Ian Brownlie, International Law at the Fiftieth Anniversary of the United Nations: General Course on Public International Law, 255 Collected Courses of The Hague Academy of International Lawes (1995). https://refer enceworks.brillonline.com/entries/the-hague-academy-collected-courses/*A9789041103185_01

International Law Association, Final Report of the Committee: Statement of Principle Applicable to the Formation of General Customary International law, Committee on Formation of Customary (General) International Law, London Conference (2000). https://www.law.umich.edu/facultyhome/drwcasebook/Documents/Document s/ILA\%20Report\%20on\%20Formation\%20of\%20Customary\%20International\%20Law.pdf

John Tasioulas, Opinio Juris and the Genesis of Custom: A Solution to the 'Paradox', 26 Australian Yearbook of International Law, n. ${ }^{\circ}$ 1, 199-205 (2007). https://doi.org/10.1163/26660229-026-01-900000008

James Crawford, The Identification and Development of Customary International Law, en Keynote Speech Delivered in the Spring Conference of the ILA, British Branch, Foundations and Futures of International Law, 23 May (2014). https://www.youtube.com/watch?v=0XBc0ZjMVSM

Jan Klabbers, The Curious Condition of Custom, 8 International Legal Theory, n. 8, 29-39 (2002). https://law.ubalt. edu/centers/cicl/publications/docs/ILT_08_2002.pdf

Jean d'Aspremont, Formalism and The Sources of International Law: A Theory of the Ascertainment of Legal Rules (Oxford University Press, 2011). https://papers.ssrn.com/sol3/papers.cfm?abstract_id=1969795

Jean d'Aspremont, The Decay of Modern Customary International Law in Spite of Scholarly Heroism, Global Community: Yearbook of International Law and Jurisprudence, 1-40 (2015). https://papers.ssrn.com/sol3/pa pers.cfm?abstract_id $=1969795$

Jean René Dupuy, Coutume sage et coutume sauvage, en Mélanges offerts à Charles Rousseau: La Communauté Internationale, 75-87 (R Jean René Dupuy ed., Pedone, 1974). http://www.sfdi.org/wp-content/uploads/201 4/03/MelRousseau1.pdf

I Lassa Francis Lawrence Oppenheim, International Law: A Treatise (Peace) (Longmans Green and Co.,1905).

Lewis Roberts, The Unwritten Law, 10 Kentucky Law Journal, n. ${ }^{\circ}$ 2, 45-52 (1922). https://uknowledge.uky.edu/klj /vol10/iss2/2/ 
Luigi Ferrari, Méthodes de Recherche de la Coutume Internationale dans la Pratique des États, 192 Collected Courses of The Hague Academy of International Law (1965). https://referenceworks.brillonline.com/entries/the-hagu e-academy-collected-courses $/{ }^{*}$ A $9789024733736 \_02$

Malcolm Shaw, International Law (6.. ed., Cambridge University Press, 2008).

Maurice Mendelson, The International Court of Justice and the Sources of International Law, en Fifty Years of the International Court of Justice, 63-89 (Vaughan Lowe \& Malgosia Fitzmaurice eds., Cambridge University Press, 1996). https://doi.org/10.1017/CBO9780511560101.007

Martti Koskenniemi, The Gentle Civilizer of Nations: The Rise and Fall of International Law 1870-1960 (Cambridge University Press, 2009). https://doi.org/10.23979/fypr.44843

I Martti Koskenniemi, Normative Force of Habit; International Custom and Social Theory, en Finnish Yearbook of International Law, 77-99 (1990).

Michael Scharf, Customary International Law in Times of Fundamental Change: Recognizing Grotian Moments (Cambridge University Press, 2013). https://doi.org/10.1017/CBO9781139649407

Michael Wood, Second report on identification of customary international law, A/CN.4/672, UN International Law Commission, Sixty-sixth Session (2014). https://digitallibrary.un.org/record/774305

W. Michael Reisman, The Cult of Custom in the Late 20th Century, 17 California Western International Law Journal, 133-145 (1987). https://digitalcommons.law.yale.edu/fss_papers/732/?utm_source=digitalcommons.law.yale .edu\%2Ffss_papers\%2F732\&utm_medium=PDF\&utm_campaign=PDFCoverPages

Michel Virally, Fuentes del Derecho Internacional, en Manual de Derecho Internacional Público (Max Sørensen ed., Fondo de Cultura Económica, 2000).

Onuma Yasuaki, A Transcivilizational Perspective on International Law: Questioning Prevalent Cognitive Frameworks in the Emerging Multi-polar and Multi-Civilizational World of the Twenty-First Century (The Hague Academy of International Law, 2010).

Onuma Yasuaki, Is the International Court of Justice and Emperor without Clothes? 8 International Legal Theory, ${ }^{\circ}{ }^{\circ} 1$, 1-10 (2002). https://law.ubalt.edu/centers/cicl/publications/docs/ILT_08_2002.pdf

I. H. E. Patient, Mistake of law-A mistake? 51 Journal of Criminal Law, n. ${ }^{\circ}$ 3, 326-337 (1987). https://doi.org/10.1 $177 / 002201838705100308$

J. Patrick Kelly, The Twilight of Customary International Law, 40 Virginia Journal of International Law, n. ${ }^{\circ}$ 2, 450-544 (2000). https://papers.ssrn.com/sol3/papers.cfm?abstract_id=1116367

Peter Elman, Mistake of Fact and Mistake of Law, 8 Israel Law Review, n. ${ }^{\circ}$ 1, 145-150 (1973). https://doi.org/10.10 17/S0021223700004027

Peter Haggenmacher, La doctrine des deux éléments du droit coutumier dans la jurisprudence de la Cour International", 90 Revue Générale du Droit International Public, 5-125 (1986).

Peter Malanczuk, Akehurst's Modern Introduction to International Law (7.. ed., Routledge, 1997).

Peter Tomka, Custom and the International Court of Justice, 12 Law and Practice of International Courts and Tribunals, n. ${ }^{\circ} 2,195-216$ (2013). https://doi.org/10.1163/15718034-12341253

P. H. Winfield, Mistake of Law, 59 Law Quarterly Review, 327-342 (1943). https://heinonline.org/HOL/LandingP age?handle=hein.journals/lqr59\&div=35\&id=\&page $=$

Committee Advisory of Jurists for the establishment of the Permanent Court of International Justice, Procès-Verbaux of the Proceedings Committee with Annexes (1920). https://www.lawbookexchange.com/pages/books/45256/league-of-nations-advisory-committee-of-ju rists/proces-verbaux-of-the-proceedings-of-the-committee-june-16th-july

Romanos 9:20, New Living Translation.

Robert Jennings, The Identification of International Law, International Law: Teaching and Practice (Bin Cheng ed., 1982). 
Rudolf Bernhardt, Custom and Treaty in The Law of the Sea, 205 Collected Courses of The Hague Academy of International Law (1987). https://referenceworks.brillonline.com/entries/the-hague-academy-collected-course s/*A9780792303220_02

Rudolf Geiger, Customary International Law in the Jurisprudence of the International Court of Justice: A Critical Appraisal, en From Bilateralism to Community Interest: Essays in Honour of Bruno Simma (Ulrich Fastenrath, Rudolf Geiger \& Daniel-Erasmus Khan eds., Oxford University Press, 2011).

Samuel Williston, Written and Unwritten Law, 17 American Bar Association Journal, n. 1, 39-41 (1931). https://w ww.jstor.org/stable/25708136

Stefan Talmon, Determining Customary International Law: The ICJ's Methodology between Induction, Deduction and Assertion, 26 European Journal of International Law, n. ${ }^{\circ} 2,417-443$ (2015). https://doi.org/10.1093/ejil/chv020

The Trial of German Major War Criminals, Speeches of the Chief Prosecutors at the Close of the Case against Individual Defendants (1946). https://www.loc.gov/rr/frd/Military_Law/pdf/NT_Vol-I.pdf

Theodor Meron, Sources of International Law, en Collected Courses of The Hague Academy, 733-736 (2003). https:/ /referenceworks.brillonline.com/entries/the-hague-academy-collected-courses/*A9789004140202_01

Tim Hillier, Sourcebook on Public International Law (Cavendish Publishing Limited, 1998).

Tullio Treves, Customary International Law, Max Planck Encyclopedia of Public International Law (2006). https://opil.ouplaw.com/view/10.1093/law:epil/9780199231690/law-9780199231690-e1393?rskey $=$ RqdNcJ\&result $=1 \&$ prd $=$ MPIL

United Nations Conference on International Organization UNIO, San Francisco, Vol. XIV (1945). https://digitalli brary.un.org/record/1300969/files/UNIO-Volume-14-E-F.pdf

William Thomas Worster, The Inductive and Deductive Methods in Customary International Law Analysis: Traditional and Modern Approaches, 45 Georgetown Journal of International Law, n. ${ }^{\circ}$ 2, 445-521 (2014). https://doi.org/ $10.2139 /$ ssrn.2197104

Yoram Dinstein, The Interaction between Customary International Law and Treaties, 322 Collected Courses of The Hague Academy of International Law (2006). https://referenceworks.brillonline.com/entries/the-hague-acade my-collected-courses $/{ }^{*}$ A9789004161016_02

\section{Notas}

* Artículo de investigación

1 Committee Advisory of Jurists for the establishment of the Permanent Court of International Justice, Procès-Verbaux of the Proceedings Committee with Annexes (1920). Las negrillas son nuestras.

2 Fabián Cárdenas, Epilogue: International Litigation and the Stamp of Post-modernity, en The Law of International Litigation: Legal Tools and Strategy for International Disputes Resolution in the 21 Century, 457-475 (Rafael Prieto Sanjuán ed., Pontificia Universidad Javeriana \& Editorial Ibáñez, 2015).

3 Aunque la Comisión se refiere al "Derecho Internacional Consuetudinario" nosotros nos referiremos a la "Costumbre Internacional”. Consideramos que en este aspecto puede haber un vacío en la traducción que la propia Comisión hace del concepto del inglés al español, el cual en los textos originales se refiere simplemente a customary law.

4 I Martti Koskenniemi, Normative Force of Habit; International Custom and Social Theory, en Finnish Yearbook of International Law, 77-99 (1990).

$5 \quad$ Estatuto de la Corte Internacional de Justicia (1945), Art. 38(b).

6 Fabián Cárdenas \& Oscar Casallas, A Large Cup of Opinio Juris and Practice to Taste: The Recipe of Contemporary Customary International Law?, 8 Colombian Yearbook of International Law, 87-130 (2015).

7 Respecto de los elementos tradicionales ver por ejemplo la descripción que se hace en Michael Scharf, Customary International Law in Times of Fundamental Change: Recognizing Grotian Moments, 29 y ss. (Cambridge University Press, 2013); Hugh Thirlway, The Sources of International Law, 53 (Oxford University Press, 2014).

8 Michel Virally, Fuentes del Derecho Internacional, en Manual de Derecho Internacional Público, 152 (Max Sørensen ed., Fondo de Cultura Económica, 2000).

9 "It was shown that there was a reluctance of States to be bound by compulsory jurisdiction without a precise framework of legal norms to be applied by such an international tribunal". Alain Pellet, The Statute of the International Court of 
Justice: A Commentary, 683, Art. 38 (Andreas Zimmermann, Christian Tomuschat \& Karin Oellers-Frahm eds., Oxford University Press, 2006).

10 Onuma Yasuaki, Is the International Court of Justice and Emperor without Clothes?, 8 International Legal Theory, n. ${ }^{\circ}$, 1-10 (2002).

11 Caso Serbian Loans, PCIJ, Judgement, Series A, Nos. 20/21 (1929).

12 Theodor Meron, Sources of International Law, en Collected Courses of The Hague Academy, 733-736 (2003).

13 Alain Pellet, Art. 38, supra nota 10.

14 Íd., 293

15 Íd., 295

16 Alain Pellet, Art. 38, supra nota 10.

17 "You must take a course amounting to refusal of justice merely because no definite convention or custom appeared. What, therefore, is the difference between my distinguished opponent and myself? He leaves the judge in a state of compulsory blindness forced to reply on subjective opinions only; l allow him to consider the cases that come before him with both eyes open". Committee Advisory of Jurists for the establishment of the Permanent Court of International Justice, ProcèsVerbaux of the Proceedings Committee with Annexes (1920).

18 Íd., 294.

19 James Crawford, The Identification and Development of Customary International Law, en Keynote Speech Delivered in the Spring Conference of the ILA, British Branch, Foundations and Futures of International Law, 23 May (2014).

20 Íd., 2.

21 Committee Advisory of Jurists for the establishment of the Permanent Court of International Justice, Procès-Verbaux of the Proceedings Committee with Annexes (1920).

22 Estatuto de la Corte Internacional de Justicia (1945), Art. 38(b).

23 United Nations Conference on International Organization UNIO, San Francisco, Vol. XIV (1945).

24 Jean d'Aspremont, The Decay of Modern Customary International Law in Spite of Scholarly Heroism, Global Community: Yearbook of International Law and Jurisprudence, 1-40 (2015).

25 Ver abajo, "Las inconsistencias de la ortodoxia".

26 Alain Pellet, Art. 38, supra nota 10.

27 Íd.

28 Curtis Bradley, A State Preference Account of Customary International Law Adjudication, SSRN (2014).

29 Christian J. Tams, The International Court as a "Law-Formative Agency", en The Development of International Law by the International Court of Justice (Christian J. Tams and James Sloan eds., Oxford University Press, 2013).

30 De hecho, el Segundo Reporte sobre la Identificación de la Costumbre Internacional afirma que el DDE es ampliamente aceptado. Véase Michael Wood, Second report on identification of customary international law, A/CN.4/672, UN International Law Commission, Sixty-sixth Session, para. 21 (2014).

31 Peter Haggenmacher, La doctrine des deux éléments du droit coutumier dans la jurisprudence de la Cour International", 90 Revue Générale du Droit International Public, 5-125 (1986).

32 Caso del S.S. Lotus (France vs. Turkey), ICJ, Judgment, PCIJ, Series A, No. 10 (1927).

33 Íd., 28.

34 Peter Tomka, Custom and the International Court of Justice, 12 Law and Practice of International Courts and Tribunals, n. 2,200 (2013).

35 Alain Pellet, Art. 38, supra nota 10.

36 Caso de la Plataforma Continental del Norte (Alemania vs. Dinamarca, Países Bajos), ICJ, Merits, ICJ Reports, para. 77 (1969); Caso del Asilo (Colombia vs. Peru), ICJ, Advisory Opinion, ICJ Reports, 266-276 (1950); Caso de S.S. Wimbledon (UK, France, Italy, Japan vs. Germany), Merits, PCIJ, Series A, No. L, 25 (1923); Caso Fisheries (UK vs. Norway), ICJ, Merits, ICJ Reports, 116-139 (1951); Caso Rights of Nationals of the USA in Morocco (France vs. US), ICJ, Merits, ICJ Reports, 176-200 (1952); Caso Right of Passage over indian Territory (Portugal vs. India), ICJ, Merits, ICJ Reports, 6, 4 (1960).

37 Caso Military and Paramilitary Activities in and against Nicaragua (Nicaragua vs. USA), ICJ, Judgment, ICJ Reports, para. 184(1986).

38 Caso Jurisdictional Immunities of the State (Germany vs. Italy, Greece Intervening), ICJ, ICJ Reports, para. 55 (2012).

39 El principio de generalidad implica la participación de "los Estados cuyos intereses se ven particularmente afectados", Casos del North Sea Continental Shelf (Federal Republic of Germany vs. Denmark, Netherlands), ICJ, Merits, ICJ Reports, 3, 42, para. 73 (1969). See also Fisheries Case (UK vs. Norway), op. cit., 116, 131; Fabián Cárdenas Castañeda \& Oscar Casallas, op. cit.

40 Malcolm Shaw, International Law, 84 (6.a ed., Cambridge University Press, 2008).

41 Corte Internacional de Justicia. Caso Asilo (Colombia vs. Peru); Malcolm Shaw, supra nota 41; Diego Uribe \& Fabián Cárdenas, Derecho Internacional Ambiental (Universidad Jorge Tadeo Lozano, 2010); Tim Hillier, Sourcebook on 
Public International Law (Cavendish Publishing Limited, 1998); Corte Internacional de Justicia. Caso Pescaderías (UK vs. Norway), 8; Peter Malanczuk, Akehurst's Modern Introduction to International Law, 43 (7.a ed., Routledge, 1997); Corte Internacional de Justicia. Caso derecho de paso por el territorio indio (Portugal vs. India), 6, 40.

42 Bin Cheng, United Nations Resolutions on Outer Space: "Instant" International Customary Law?, 5 Indian Journal of International Law, 23-112 (1965); Diego Mejía-Lemus, Some Considerations regarding "Instant International Customary Law”, Fifty Years Later, Indian Journal of International Law, n. ${ }^{\circ} 55,85-108$ (2015).

43 "[...] with respect to the other element usually regarded as necessary before a conventional rule of international law, it might be that, even without: the passage of any considerable period of time, a very widespread and representative participation in the convention might suffice of itself, provided it included that of States whose interest were specially affected'”. Casos del North Sea Continental Shelf(Federal Republic of Germany vs. Denmark, Netherlands), ICJ, Merits, ICJ Reports, 3, 42, para. 73 (1969).

44 Fabián Cárdenas \& Oscar Casallas, op. cit.

45 Malcolm Shaw, op. cit., 104.

46 Yoram Dinstein, The Interaction between Customary International Law and Treaties, 322 Collected Courses of The Hague Academy of International Law, 72 (2006).

47 Anthony D’Amato, Trashing Customary International Law, 81 American Journal of International Law, 101-105 (1987).

48 Fabián Cárdenas Castañeda \& Oscar Casallas, op. cit.

49 Una variedad de casos ha afirmado que cuando una norma consuetudinaria va a ser establecida "tiene que dirigir su atención a la práctica y la opinio juris de los Estados", véase Caso Military and Paramilitary Activities in and against Nicaragua (Nicaragua vs. USA), ICJ, Judgment, ICJ Reports,para. 183 \& 211 (1986). Caso Asilo (Colombia vs. Perú), pp. 276-277; Caso derecho de paso por territorio indio (Portugal v. India), Casos del North Sea Continental Shelf (Federal Republic of Germany vs. Denmark, Netherlands), ICJ, Merits, ICJ Reports, para. 77 (1969); Caso Legality of the Threat or Use of Nuclear Weapons, ICJ, Advisory Opinion, ICJ Reports, paras. 65-73 (1996).

50 Íd.

51 Estatuto de la Corte Internacional de Justicia (1945), Art. 38(b).

52 James Crawford, op. cit.

53 Onuma Yasuaki, supra nota 11.

54 Caso Accordance with International Law of the Unilateral Declaration of Independence in Respect of Kosovo, ICJ, Advisory Opinion, ICJ Reports, paras. 79-84 (2010). Corte Internacional de Justicia. Accordance with International Law of the Unilateral Declaration of Independence in Respect of Kosovo, Advisory Opinion, ICJ Reports, paras. 79-84 (2010)

55 Onuma Yasuaki, supra nota 11.

56 Íd., 5.

57 Íd., 4.

58 Onuma Yasuaki, A Transcivilizational Perspective on International Law: Questioning Prevalent Cognitive Frameworks in the Emerging Multi-polar and Multi-Civilizational World of the Twenty-First Century, 220-224 (The Hague Academy of International Law, 2010).

59 Maurice Mendelson, The International Court of Justice and the Sources of International Law, en Fifty Years of the International Court of Justice, 67 (Vaughan Lowe \& Malgosia Fitzmaurice eds., Cambridge University Press, 1996).

60 La búsqueda por CI en la CIJ nunca ha sido consistente "This being so, it is indeed not certain that the Court's approach for finding customary rules evidencing general practice accepted as law has always been as rigorous as it would have been, even within the large margin of appreciation implied by such a definition" Alain Pellet, op. cit., 706. En la misma corriente, ver Rudolf Geiger, Customary International Law in the Jurisprudence of the International Court of Justice: A Critical Appraisal, en From Bilateralism to Community Interest: Essays in Honour of Bruno Simma (Ulrich Fastenrath, Rudolf Geiger \& Daniel-Erasmus Khan eds., Oxford University Press, 2011). También conviene consultar, Stefan Talmon, Determining Customary International Law: The ICJ's Methodology between Induction, Deduction and Assertion, 26 European Journal of International Law, n. ${ }^{\circ}$ 2, 417-443 (2015).

61 Íd., 760.

62 Art. 38 del Estatuto de la CIJ.

63 Humphrey Waldock, General Course on Public International Law, 106 Collected Courses of The Hague Academy of International Law, 41 (1962).

64 Hersch Lauterpacht, Régles Générales du Droit de la Paix, 62 Collected Courses of The Hague Academy of International Law, 45 (1937).

65 I Lassa Francis Lawrence Oppenheim, International Law: A Treatise (Peace), 22 (Longmans Green and Co., 1905).

66 Luigi Ferrari, Méthodes de Recherche de la Coutume Internationale dans la Pratique des États, 192 Collected Courses of The Hague Academy of International Law, 243 (1965).

67 Curtis Bradley, op. cit., 7-8. 
68 I. H. E. Patient, Mistake of law- A mistake?, 51 Journal of Criminal Law, n. ${ }^{\circ} 3,337$ (1987); Peter Elman, Mistake of Fact and Mistake of Law, 8 Israel Law Review, n. ${ }^{\circ}$ 1, 145-150 (1973); P. H. Winfield, Mistake of Law, 59 Law Quarterly Review, 327-342 (1943) ; Dan Being, Mistake of Law, 30 Israel Law Review, n. ${ }^{\circ}$ 1-2, 214-222 (1996); Arzt Gunther, Ignorance or Mistake of Law, 24 American Journal of Comparative Law, n. ${ }^{\circ} 4,646-679$ (1976).

69 Curtis Bradley, op. cit., 10.

70 "I propose that opinio juris be interpreted as a requirement that states generally believe that it is desirable now or in the near future to have an authoritative legal principle or rule prescribing, permitting, or prohibiting certain state conduct". Brian D. Lepard, Customary International Law: A New Theory with Practical Applications, 97-98 (Cambridge University Press, 2010).

71 Ver una posible solución de la paradoja en John Tasioulas, Opinio Juris and the Genesis of Custom: A Solution to the 'Paradox', 26 Australian Yearbook of International Law, n. ${ }^{\circ}$ 1, 199-205 (2007). Ver, por ejemplo, Daniel Harold Levine, The Chronological Paradox in Customary International Law or, the Virtue of Sloopy Timing in a Messy World, (2005) (Ph.D. Dissertation, Georgetown University).

72 Como un ejemplo, véase el proyecto TRICI Law y The Theory \& Philosophy of Customary International Law and its Interpretation, evento académico llevado a cabo el 24 y 25 de mayo de 2019, en la Universidad de Groningen, Países Bajos.

73 International Law Association, Final Report of the Committee: Statement of Principle Applicable to the Formation of General Customary International law, Committee on Formation of Customary (General) International Law, London Conference, 8 (2000).

74 Para otra explicación sobre los matices de dichos verbos ver Brian D. Lepard, op. cit., 112-121.

75 Anthony D’Amato, New Approaches to Customary International Law, 105 American Journal of International Law, n. ${ }^{\circ} 1,163-170$ (2011).

76 Anthony D’Amato, Customary International Law: A Reformulation, 4 International Legal Theory, n. ${ }^{\circ}$ 1, 1-6.

77 Íd., 3.

78 François Rigaux, Hans Kelsen on International Law, 9 European Journal of International Law, n. ${ }^{\circ}$ 2, 325-343 (1998); Danilo Zolo, Hans Kelsen: International Peace through International Law, 9 European Journal of International Law, 206-324 (1998).

79 El uso de la palabra según la RAE señala que es la manera habitual de actuar o comportarse "habit of doing or does regularly”.

80 Grigory Tunkin, International Law in the International System, 147 Collected Courses of The Hague Academy of International Law (1975).

81 Michael Scharf, op. cit.

82 Hugo Grotius, The Freedom of the Seas : Or the Right Which Belongs to the Dutch to Take Part in the East Indian Trade, 12 (Oxford University Press, 1916).

83 Íd., 39.

84 Romanos 9:20, New Living Translation. "No, don't say that. Who are you, a mere human being, to argue with God? Should the thing that was created say to the one who created it, "Why have you made me like this?"

85 Alfred Verdross, Les Principes Généraux du Droit dans la Jurisprudence Internationale, 52 Collected Courses of The Hague Academy of International Law (The Hague Academy of International Law,1935).

86 The Trial of German Major War Criminals, Speeches of the Chief Prosecutors at the Close of the Case against Individual Defendants (1946). Ver también la Declaración Universal de los Derechos Humanos (1948) “[...] Whereas disregard and contempt for human rights have resulted in barbarous acts which have outraged the conscience of mankind, and the advent of a world in which human beings shall enjoy freedom of speech and belief and freedom from fear and want has been proclaimed as the highest aspiration of the common people $[. .$.$] ”.$

87 Martti Koskenniemi, The Gentle Civilizer of Nations: The Rise and Fall of International Law 1870-1960 (Cambridge University Press, 2009).

88 Íd., 398.

89 Brian D. Lepard, op. cit.

90 Íd.

91 Íd.

92 Rudolf Bernhardt, Custom and Treaty in The Law of the Sea, 205 Collected Courses of The Hague Academy of International Law, 266 (1987).

93 Ian Brownlie, International Law at the Fiftieth Anniversary of the United Nations: General Course on Public International Law, 255 Collected Courses of The Hague Academy of International Lawes (1995).

94 Fabián Cárdenas \& Oscar Casallas. op. cit., 94.

95 El concepto no es nuevo, ver, por ejemplo, Lewis Roberts, The Unwritten Law, 10 Kentucky Law Journal, n. ${ }^{\circ} 2,45-52$ (1922); Samuel Williston, Written and Unwritten Law, 17 American Bar Association Journal, n. $1,39-41$ (1931).

96 Humphrey Waldock, op. cit., 39-42. 
97 Martti Koskenniemi, supra nota 87.

98 Jean René Dupuy, Coutume sage et coutume sauvage, en Mélanges offerts à Charles Rousseau: La Communauté Internationale, 75-87 (R Jean René Dupuy ed., Pedone, 1974).

99 Hugh Thirlway, Concepts, Principles, Rules and Analogies: International and Municipal Legal Reasoning, 294 Collected Courses of The Hague Academy of International Law (2002).

100 Helbert Hart, The Concept of Law, 92 (2.a ed., Clarendon Press, 1994).

101 Bin Cheng. op. cit., 23-112; Mejía-Lemus, op. cit., 85.

102 La RAE define proceso como conjunto de las fases sucesivas de un fenómeno natural o de una operación artificial.

103 Tullio Treves, Customary International Law, Max Planck Encyclopedia of Public International Law (2006).

104 Jean d'Aspremont, Formalism and The Sources of International Law: A Theory of the Ascertainment of Legal Rules, 170 (Oxford University Press, 2011).

105 Íd., 162.

106 Íd., 164.

107 Íd.

108 Jan Klabbers, The Curious Condition of Custom, 8 International Legal Theory, n. ${ }^{\circ}$ 8, 29-39 (2002).

109 William Thomas Worster, The Inductive and Deductive Methods in Customary International Law Analysis: Traditional and Modern Approaches, 45 Georgetown Journal of International Law, n. ${ }^{\circ}$ 2, 445-521 (2014).

110 J. Patrick Kelly, The Twilight of Customary International Law, 40 Virginia Journal of International Law, n. ${ }^{\circ} 2,528$ (2000).

111 Robert Jennings, The Identification of International Law, International Law: Teaching and Practice, 3-6 (Bin Cheng ed., 1982).

112 David Fidler, Challenging the Classical Concept of Custom: Perspectives on the Future of Customary International Law, 39 German Yearbook of International Law, n. 198,224 (1996).

113 Onuma Yasuaki, supra nota 11.

114 W. Michael Reisman, The Cult of Custom in the Late 20th Century, 17 California Western International Law Journal, 133 (1987).

115 Íd.

116 Véase los reportes A/CN.4/663; A/CN.4/672*; A/CN.4/682; A/CN.4/695; A/CN.4/717; y los memorándums A/ CN.4/659; A/CN.4/691*; A/CN.4/710/Rev.1

117 Comisión de Derecho Internacional de la ONU, CDI, Identification of customary international law. A/RES/73/203 (2019).

118 Comisión de Derecho Internacional de la ONU, Draft conclusions on identification of customary international law, with commentaries. A/73/10, 122 (2018).

119 Íd., 124.

120 Comisión de Derecho Internacional de la ONU, Second report on identification of customary international law, A/ CN.4/672, 8 (2014).

121 Íd., 3.

122 Comisión de Derecho Internacional de la ONU, Draft conclusions on identification of customary international law, with commentaries, A/73/10, 122 (2018).

123 "Practice without acceptance as law (opinio juris), even if widespread and consistent, can be no more than a non-binding usage, while a belief that something is (or ought to be) the law unsupported by practice is mere aspiration; it is the two together that establish the existence of a rule of customary international law." Comisión de Derecho Internacional de la ONU, Draft conclusions on identification of customary international law, with commentaries, A/73/10, 126 (2018).

124 Comisión de Derecho Internacional de la ONU, Draft conclusions on identification of customary international law, with commentaries, A/73/10, 122 (2018).

125 Casos del North Sea Continental Shelf (Federal Republic of Germany vs. Denmark, Netherlands), supra nota 2, 3, 42.

126 Íd., 74.

127 Caso Military and Paramilitary Activities in and against Nicaragua (Nicaragua vs. USA), ICJ, Judgment, ICJ Reports, para. 188 (1986).

128 Casos del North Sea Continental Shelf (Federal Republic of Germany vs. Denmark, Netherlands), ICJ, Merits, ICJ Reports, 77 (1969).

129 Comisión de Derecho Internacional de la ONU, op. cit., 138 (2018).

130 Caso del S.S. Lotus (France vs. Turkey), supra nota 33.

131 Disponibles en: http://legal.un.org/ilc/guide/1_13.shtml\#top

132 Como lo son Cuba y El Salvador, de acuerdo a la información suministrada a la Comisión de Derecho Internacional como insumo al trabajo del relator especial. 
133 En donde se pueden agrupar Botsuana y El Salvador, de acuerdo a la información suministrada a la Comisión de Derecho Internacional como insumo al trabajo del relator especial.

134 Esta reflexión sobre la costumbre como producto de campañas de argumentación -que aquí no se discute-ha sido explicada en Fabián Cárdenas, Custom as the product of successful argumentative campaigns, en International Organizations, Non-State Actors and the Formation of Customary International Law (S. Droubi \& J. d'Aspremont eds., Manchester University Press, 2020). Su construcción inicial fue planteada en Fabián Cárdenas, Customary International Law as an Argumentative Framework (2016) (tesis doctoral, Pontificia Universidad Javeriana).

135 Fabián Cárdenas \& Santiago Rojas, Costumbre internacional: una lectura desde Latinoamérica a la perspectiva ortodoxa de la fuente no escrita del derecho internacional, en Manual de Derecho Internacional Público (René Urueña ed., Universidad de Los Andes, 2020).

136 Respecto de la comunidad de abogados internacionalistas como agentes validadores del derecho internacional, véase Fabián Cárdenas \& Jean d'Aspremont, Epistemic Communities in International Adjudication, en Max Planck Encyclopedia of International Procedural Law (2020).

\section{Licencia Creative Commons CC BY 4.0}

Para citar este artículo/To cite this article: Fabián Cárdenas, ¿Un caso de "volver al 'futuro" ?: Las conclusiones sobre la identificación del derecho internacional consuetudinario de la Comisión de Derecho Internacional de la ONU, 69 Vniversitas (2020). https://doi.org//10.11144/Javeriana.vj69.cvfc 